\title{
ASTEROID BELTS IN DEBRIS DISK TWINS: VEGA AND FOMALHAUT
}

\author{
Kate Y. L. Su${ }^{1}$, George H. Rieke ${ }^{1}$, Renu Malhotra ${ }^{2}$, Karl R. Stapelfeldt ${ }^{3}$, A. Meredith Hughes ${ }^{4}, 10$, Amy Bonsor $^{5}$, \\ David J. Wilner ${ }^{6}$, Zoltan Balog ${ }^{7}$, Dan M. Watson ${ }^{8}$, Michael W. Werner ${ }^{9}$, and Karl A. Misselt ${ }^{1}$ \\ ${ }^{1}$ Steward Observatory, University of Arizona, 933 N Cherry Avenue, Tucson, AZ 85721, USA; ksu @ as.arizona.edu \\ ${ }^{2}$ Lunar and Planetary Laboratory, University of Arizona, Tucson, AZ 85721, USA \\ ${ }^{3}$ Code 667, NASA Goddard Space Flight Center, Greenbelt, MD 20771, USA \\ ${ }^{4}$ Department of Astronomy, University of California, Berkeley, CA 94720, USA \\ ${ }^{5}$ UJF-Grenoble 1/CNRS-INSU, Institut de Planétologie et d'Astrophysique de Grenoble (IPAG), UMR 5274, Grenoble, F-38041, France \\ ${ }^{6}$ Harvard-Smithsonian Center for Astrophysics, 60 Garden Street, Cambridge, MA 02138, USA \\ ${ }^{7}$ Max-Planck-Institut für Astronomie, Königstuhl 17 D-69117, Heidelberg, Germany \\ ${ }^{8}$ Department of Physics and Astronomy, University of Rochester, Rochester, NY 14627, USA \\ ${ }^{9}$ JPL/Caltech, 4800 Oak Grove Drive, Pasadena, CA 91109, USA \\ Received 2012 October 19; accepted 2012 December 6; published 2013 January 16
}

\begin{abstract}
Vega and Fomalhaut are similar in terms of mass, ages, and global debris disk properties; therefore, they are often referred to as "debris disk twins." We present Spitzer 10-35 $\mu \mathrm{m}$ spectroscopic data centered at both stars and identify warm, unresolved excess emission in the close vicinity of Vega for the first time. The properties of the warm excess in Vega are further characterized with ancillary photometry in the mid-infrared and resolved images in the far-infrared and submillimeter wavelengths. The Vega warm excess shares many similar properties with the one found around Fomalhaut. The emission shortward of $\sim 30 \mu \mathrm{m}$ from both warm components is well described as a blackbody emission of $\sim 170 \mathrm{~K}$. Interestingly, two other systems, $\epsilon$ Eri and HR 8799, also show such an unresolved warm dust using the same approach. These warm components may be analogous to the solar system's zodiacal dust cloud, but of far greater mass (fractional luminosity of $\sim 10^{-5}$ to $10^{-6}$ compared to $10^{-8}$ to $10^{-7}$ ). The dust temperature and tentative detections in the submillimeter suggest that the warm excess arises from dust associated with a planetesimal ring located near the water-frost line and presumably created by processes occurring at similar locations in other debris systems as well. We also review the properties of the $2 \mu \mathrm{m}$ hot excess around Vega and Fomalhaut, showing that the dust responsible for the hot excess is not spatially associated with the dust we detected in the warm belt. We suggest it may arise from hot nano grains trapped in the magnetic field of the star. Finally, the separation between the warm and cold belt is rather large with an orbital ratio $\gtrsim 10$ in all four systems. In light of the current upper limits on the masses of planetary objects and the large gap, we discuss the possible implications for their underlying planetary architecture and suggest that multiple, low-mass planets likely reside between the two belts in Vega and Fomalhaut.
\end{abstract}

Key words: circumstellar matter - infrared: stars - planetary systems - stars: individual (Vega, Fomalhaut)

Online-only material: color figures

\section{INTRODUCTION}

Debris disks were discovered by $I R A S$ as infrared excess emission arising from systems of particles analogous to enhanced Kuiper belts. They are tenuous dusty disks sustained by cometary activity and planetesimal collisions, which initiate cascades of further collisions that break bodies down into fine dust (Dominik \& Decin 2003). The dust is lost relatively quickly through photon pressure, Poynting-Robertson (P-R), or stellarwind drag forces, generally in a timescale much shorter than $10^{4}$ yr. Thus, a debris-disk-generated infrared excess requires the presence of colliding planetesimals, and a larger object (can be as small as Pluto-size) to stir them, i.e., some form of planetary system (Wyatt 2008). The large emitting area of debris makes these disks detectable through infrared/submillimeter thermal emission or optical scattered light, providing insights into the nature of unseen parent-body populations and massive perturbers around other stars.

The dust around a host star absorbs stellar radiation and re-emits in the infrared at equilibrium temperatures, balanced between absorption and emission. Consequently, the spectral

\footnotetext{
${ }^{10}$ Miller Fellow.
}

energy distribution (SED) of the infrared excesses and its relation to the dust temperatures can be used to infer the number of emitting grains and their distances from the heating star. For example, excesses in the near-infrared near $2 \mu \mathrm{m}$ should be dominated by dust at $\sim 1500 \mathrm{~K}$, excesses in the mid-infrared near $24 \mu \mathrm{m}$ should be dominated by dust at $\sim 120-150 \mathrm{~K}$, while excesses in the far-infrared are mostly from dust at $\sim 50 \mathrm{~K}$. Furthermore, excess emission at shorter wavelengths also contributes excess at longer wavelengths as Rayleigh-Jeans (a steep function of wavelengths).

Identifying excess emission around a star requires a good knowledge of stellar photospheric properties and extrapolation to long wavelengths. Positive identifications of excesses at long wavelengths are easier than at shorter wavelengths where the host star dominates, unless the signal of the star can be filtered out through interferometric techniques. There is also a concern whether excesses at mid-infrared and far-infrared are tracing separate components since the majority of debris disks are unresolved. For nearby systems, modern facilities like Spitzer and Herschel have sufficient resolution to resolve the detailed structures of the disk and reveal the complexity in disk structures in a few cases. The identification of a warm excess in these resolved systems requires precise subtraction of the stellar 
photosphere in the resolved images, which has been done for the Fomalhaut (Stapelfeldt et al. 2004), $\epsilon$ Eri (Backman et al. 2009), and HR 8799 (Su et al. 2009) systems. Ironically, some of the nearby resolved systems are saturated in the mid-infrared, making the recognition of such a component very challenging.

In this paper, we present mid-infrared spectroscopic studies centered at the two nearby A-type stars Vega and Fomalhaut obtained with the Spitzer Infrared Spectrograph (IRS) instrument and we identify warm excess emission in the vicinity of the star, clearly separated from the much brighter cold planetesimal belts that dominate the far-infrared emission. We suggest the presence of a planetesimal belt near the water-frost line of the Vega system for the first time. We compare its properties with the warm excess around Fomalhaut (Stapelfeldt et al. 2004). We discuss the properties of the warm excesses complemented with resolved infrared and submillimeter images of both systems. Two other spatially resolved debris systems, $\epsilon$ Eri (Backman et al. 2009) and HR 8799 (Su et al. 2009), are also known to possess a similar warm belt that is fainter and clearly separated from the more prominent cold belt using a similar approach. These warm components may be analogous to our asteroid belt, but of far greater mass. We discuss the implications and origins of this two-belt configuration in light of the similarity in dust temperature distribution found around unresolved debris systems between solar-like and early-type stars by Morales et al. (2011).

The paper is organized as follows. The observations and data reduction are described in Section 2, including both Spitzer IRS spectroscopy and Herschel PACS imaging. Photospheric determination using ancillary data is given in Section 3.1, where we conclude that no significant excess is found from 2.2 to $8 \mu \mathrm{m}$ for both systems. Using the PACS 70 and $160 \mu \mathrm{m}$ images, we estimate the excess flux of the unresolved source centered at the star position for Vega in Section 3.2 and Section 3.3 for Fomalhaut. We construct the SEDs of the unresolved excess components along with other mid-infrared and submillimeter measurements, and suggest that they arise from a planetesimal belt placed near the water-frost line in Section 4. In Section 5, we discuss the implication of the two-belt systems on the underlying planetary configuration and provide a possible explanation for the $2 \mu \mathrm{m}$ excess in the Vega system. Conclusions are given in Section 6.

\section{OBSERVATIONS AND DATA REDUCTION}

\subsection{Spitzer IRS Spectroscopy}

To avoid saturation, we only used data taken in the IRS highresolution $(\mathrm{R} \sim 600)$ modules (short-high $(\mathrm{SH}): 9.9-19.6 \mu \mathrm{m}$ and long-high (LH): 18.7-37.2 $\mu \mathrm{m})$. The sizes of the slits are $4^{\prime \prime} .7 \times 11^{\prime \prime} .3$ and $11^{\prime \prime} .1 \times 22$. 3 for the SH and LH modules, respectively; a significant fraction of the light from a point source is outside the slit. Standard slit loss correction for a point source was applied to the extracted spectra; therefore, the part of the spectrum where a point source dominates the emission has the correct spectral shape.

IRS SH and LH spectral mapping data centered at the position of Vega were obtained through IRS calibration programs (PID $1406,1409,1411$, and 1413) in 2004. Here, we present six sets of observations where the slit was placed on the Vega position based on PCRS pointing information (no IRS peakup). These data were taken in the spectral mapping mode with two positions parallel to the slit $\left(7^{\prime \prime} .47 \mathrm{step}^{-1}\right)$ and three positions perpendicular to the slit direction $\left(1^{\prime \prime} \mathrm{step}^{-1}\right)$; we only used the

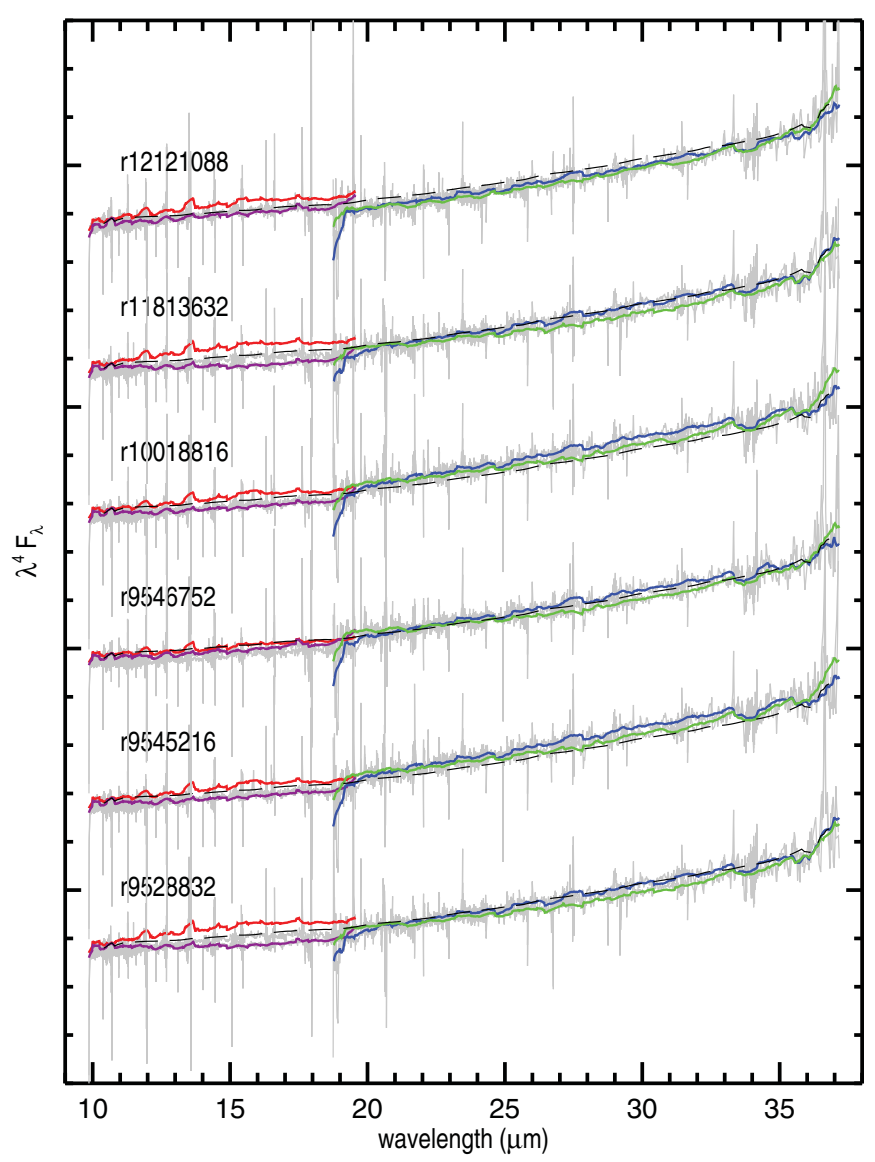

Figure 1. IRS spectra of Vega shown in $\lambda$ vs. $\lambda^{4} F_{\lambda}$ format so that a Rayleigh-Jeans spectrum is flat. Each observation (AOR) is shifted vertically for clear viewing. The unsmoothed, unscaled extracted spectra are shown in gray lines, and the scaled, smoothed spectra are shown in colors: red and purple for SH while blue and green for LH. The final combined spectrum (black dashed line) is also shown in all six AORs for comparison.

(A color version of this figure is available in the online journal.)

ones taken at the center perpendicular position (two positions along the slit center). We used the SMART software (v.8.2.5; Higdon et al. 2004) to reduce the BCD products from the Spitzer Science Center (SSC) pipeline version of S18.18. Each of the spectra was extracted with the full-slit mode without sky subtraction, flux calibrated to a point source using the S18.18 calibration, and shown in Figure 1. We then pinned all of the SH spectra to $35.03 \mathrm{Jy}$ at $10.6 \mu \mathrm{m}$ according to the absolute calibration scale defined in Rieke et al. (2008), and shifted the corresponding LH spectra (a scale factor of 0.975) to join smoothly with the SH ones. The final spectrum was obtained as a weighted average of these six AORs (black dashed line in Figure 1).

The Fomalhaut system was observed with the Spitzer IRS instrument in program PID 90 in 2004 June and November. Again, we only report here the observations with the IRS SH and LH modules, due to saturation of the signal in the lowresolution modules. In IRS SH, we obtained a standard staringmode nodded observation, with an on-target integration time of 503 s. This observation was preceded by a high-accuracy pointing peakup on a nearby star with no infrared excess, HD 216922, using the IRS Blue (13-19 $\mu \mathrm{m})$ peakup camera. With IRS LH, we obtained spectral-mapping observations with a strip of nine slit positions separated in the dispersion direction by 4 . 8 and centered on Fomalhaut. The integration time per 

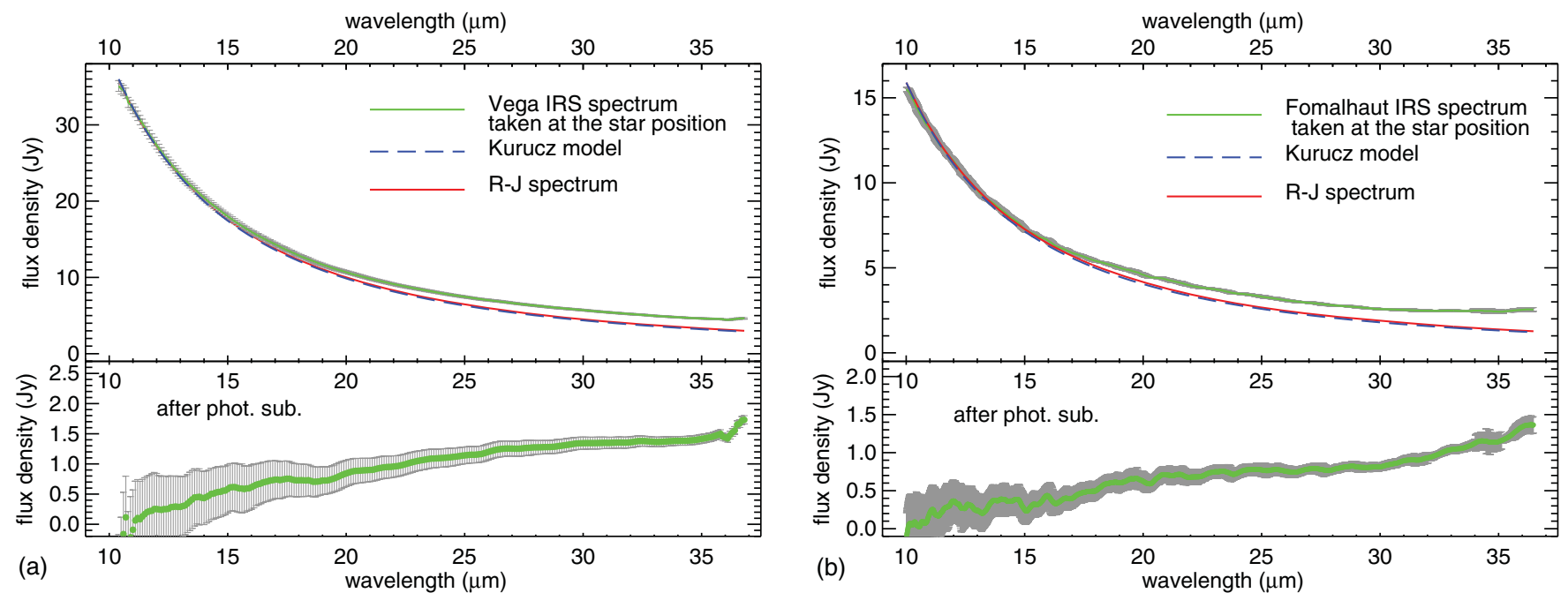

Figure 2. Spitzer IRS spectra centered at the star position for Vega (upper panel (a)) and Fomalhaut (upper panel (b)). For comparison, Kurucz atmospheric models (long dashed lines) and Rayleigh-Jeans spectra (sold red lines) are also shown. The excess spectra (after photospheric subtraction) are shown in the lower panels of the plots. The errors for these excess spectra include $2 \%$ uncertainty from the photospheric models.

(A color version of this figure is available in the online journal.)

slit position was $122 \mathrm{~s}$. The spectral mapping exercise was preceded by a moderate-accuracy IRS pointing peakup using the red $(19-26 \mu \mathrm{m})$ camera and HD 216922. Spectra at the extremes of the spectral map indicated that sky emission is negligible compared to that by Fomalhaut, so no sky subtraction was performed on the center-position spectrum we discuss here. Data reduction began with basic calibrated data products of the IRS data pipeline, version S11. From these data, we removed, by interpolation in the spectral direction, permanently bad and "rogue" pixels identified in the IRS dark-current data for all observing campaigns up to and including the one in which each Fomalhaut spectrum was taken. Again, we used SMART for full-slit extractions of spectra from the two-dimensional data. Similar observations were made of $\alpha$ Lac (A1 V) in IRS SH and $\gamma$ Dra (K1 III). Along with template spectra for these stars provided by M. Cohen (2004, private communication), we used these observations to construct relative spectral response functions (RSRFs) for each spectrometer, and in turn used these RSRFs to calibrate the Fomalhaut spectra. We estimate the resulting spectrophotometric accuracy to be approximately 5\%.

The final combined, smoothed spectra for both systems are shown in Figure 2. For comparison, the stellar photospheric models (details see Section 3.1) are also shown. Given the geometry of the outer cold rings (radius of $20^{\prime \prime}$ in the Fomalhaut system (Acke et al. 2012) and radius of $11^{\prime \prime}$ in the Vega system (Sibthorpe et al. 2010)) and the sizes of the point spread functions (PSFs) in the IRS wavelengths, the spectral flux beyond $\sim 30 \mu \mathrm{m}$ is partially contaminated by the cold ring. The spectral shape and flux level shortward of $\sim 30 \mu \mathrm{m}$ are mostly from the star and any unresolved inner warm component. This is consistent with the fact that the observed spectrum between 10 and $13 \mu \mathrm{m}$ agrees well with the expected stellar photosphere. The excess spectrum (after stellar photospheric subtraction) is also shown in the lower panel for both systems in Figure 2.

\subsection{Herschel PACS Imaging}

Herschel PACS 70 and $160 \mu \mathrm{m}$ data for Vega and Fomalhaut were obtained by the GT program and published in an early reduction by Sibthorpe et al. (2010) and Acke et al. (2012), respectively. We retrieved the archival data and reduced them with the Herschel Interactive Processing Environment (HIPE, V9.0 user release; Ott 2010). We applied the standard processing steps up to the level 1 stage. During this process, we applied 2nd level deglitching to remove outliers with "timeordered" option and $20 \sigma$ threshold. ${ }^{11}$ This is very effective for data with high levels of coverage. After producing level 1 data, we selected the science frames from the time-line by applying spacecraftspeed selection criteria (between $8^{\prime \prime} \mathrm{s}^{-1}$ and $12^{\prime \prime} \mathrm{s}^{-1}$ for the slow scan, and $18^{\prime \prime} \mathrm{s}^{-1}$ and $22^{\prime \prime} \mathrm{s}^{-1}$ for the median scan). The final level 2 mosaics were generated using highpass filtering with the script "photProject" and a pixel scale of 1 " at $70 \mu \mathrm{m}$ and $2^{\prime \prime}$ at $160 \mu \mathrm{m}$. To avoid flux loss in the highpass filtering process, we applied a circular mask of $60^{\prime \prime}$ radius centered on the position of the target. Since the PACS data on Vega and Fomalhaut were obtained with different scan rates $\left(10^{\prime \prime} \mathrm{s}^{-1}\right.$ and $20^{\prime \prime} \mathrm{s}^{-1}$, respectively), PSF observations matched to the observing parameters should be used for comparison. We used PACS data on $\alpha$ Boo (ObsId 1342247634 and 1342247635) and $\alpha$ Tau (ObsId 1342214211 and 1342214212) as our PSF reference and reduced them using the same reduction procedure including the masking radius. These two stars are ones of the PACS primary calibrators, where their fluxes and PSF behaviors are characterized by Müller et al. (2011). We have made sure that these PSF stars have the consistent encircled energy fraction as a function of circular aperture radius derived by the PACS point-source calibration (Müller et al. 2011). To illustrate the major features seen in the PACS images, we show the final $70 \mu \mathrm{m}$ mosaics of Vega and Fomalhaut in Figure 3 along with the comparison PSFs.

\section{PHOTOSPHERIC PROPERTIES AND THE IDENTIFICATION OF THE CENTRAL UNRESOLVED DISK}

In this section, we first review all ancillary photometry to establish the fidelity of the photospheric spectrum for Vega and Fomalhaut. We then assess whether the excess emission detected

\footnotetext{
11 Details about the timeordered option can be found in the HIPS documentation under the PACS photometry data reduction, http://herschel.esac.esa.int/hcss-doc-9.0/
} 

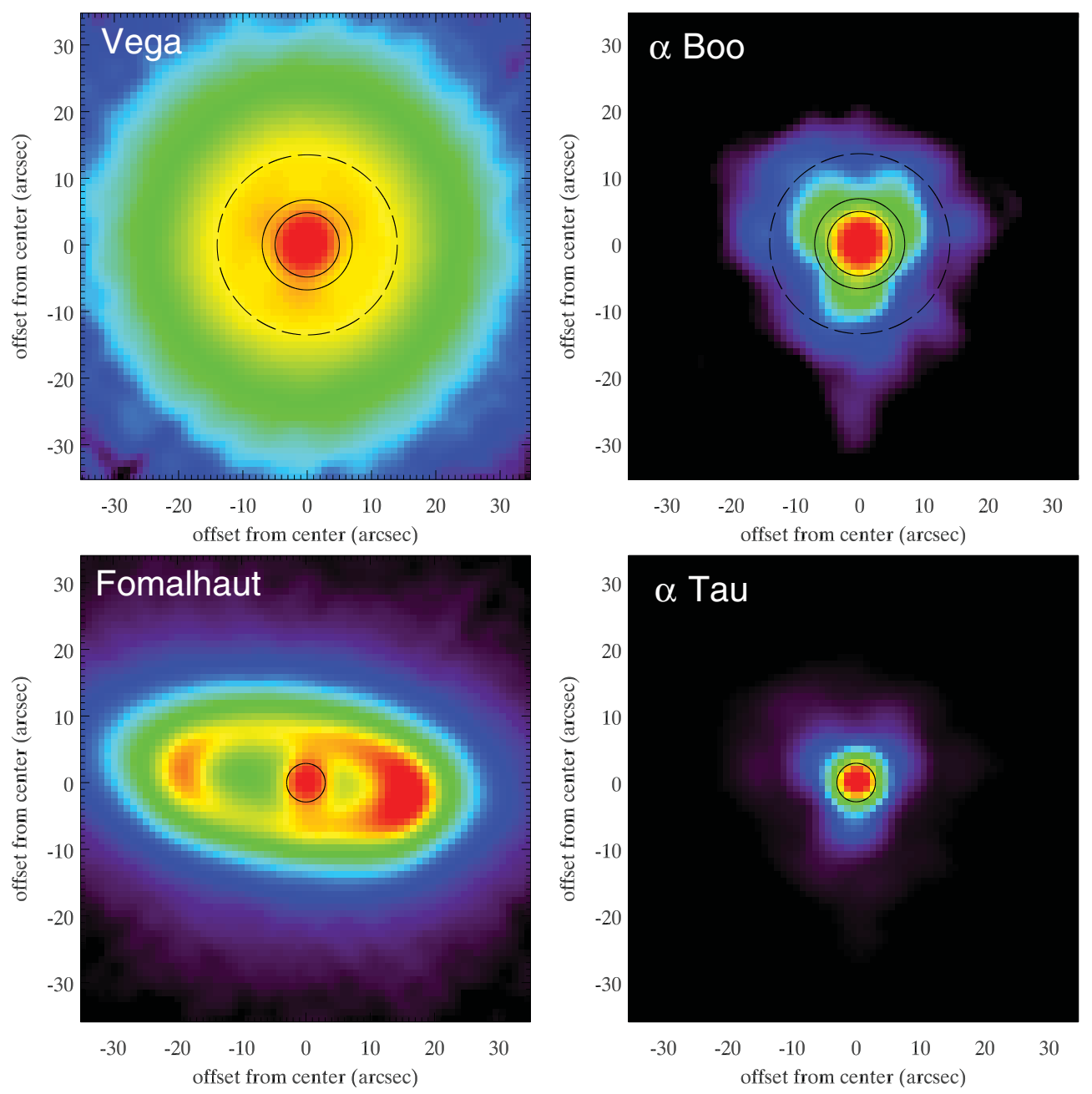

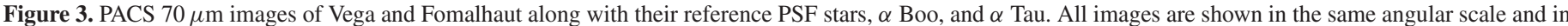

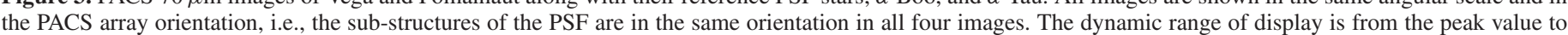

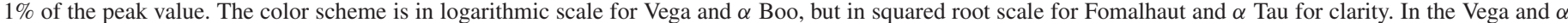

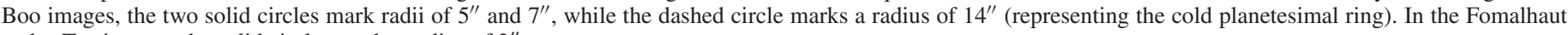
and $\alpha$ Tau images, the solid circles mark a radius of $3^{\prime \prime}$.

(A color version of this figure is available in the online journal.)

in the IRS spectrum is also detected in the resolved Herschel PACS 70 and $160 \mu \mathrm{m}$ images. Since the stellar photospheres dominate the emission in the central part of the resolved images at $70 \mu \mathrm{m}$ where the systems are best resolved, care must be taken in estimating the contribution of a central dust component without involving any further assumption of modeling. We do this in two ways: (1) with photometry using small apertures that exclude spatially extended emission and (2) with PSF subtraction using reference stars to minimize the residuals at the star position. The first approach provides an estimate of the maximum error on the central component due to contamination from other dust emission located in a more spatially extended distribution. The second approach provides a more accurate estimate of the flux of an unresolved source. In both systems, the PACS $160 \mu \mathrm{m}$ images provide only upper limits because the lower angular resolution at this longer wavelength makes it difficult to spatially differentiate the components.

\subsection{Photospheric Determination Using Ancillary Photometry}

The emission of the stellar photosphere from optical to mid-IR $(\sim 8 \mu \mathrm{m})$ was determined in a number of steps. Most infrared photometry measurements (like Spitzer/IRAC and Akari) are referred to Vega, although Vega is unsatisfactory as a standard (i.e., fast-rotating, infrared excess). However, these missions also measured Sirius using the same technique, so we have taken the measurements with nominal uncertainties of $0.01 \mathrm{mag}$ directly compared to Sirius in terms of magnitude differences. Sirius (A1V) is very similar in spectral type to Vega (A0V) and Fomalhaut (A4V), and is well behaved in the infrared with no evidence for an infrared excess (Price et al. 2004).

All three stars are severely saturated in the Two Micron All Sky Survey (2MASS) data. Therefore, for accurate measurements at $2.2 \mu \mathrm{m}$, we used data from the DIRBE instrument on COBE. The reduction of these data is described by Price et al. (2010) for analyzing stellar variability. Due to the large DIRBE beam $\left(42^{\prime} \times 42^{\prime}\right)$, the contribution from stars in the field surrounding the target of interest needs to be removed. We evaluated this effect using 2MASS data, making Sirius fainter by 0.014 mag and Fomalhaut fainter by 0.003 mag while the contribution in the Vega field is negligible. Both Vega and Fomalhaut are reported to have $K$ band excess at $1.29 \% \pm 0.19 \%$ (Absil et al. 2006) and $0.88 \% \pm 0.12 \%$ (Absil et al. 2009) above the photosphere using interferometry. After accounting for these 
$K$-band excesses of Vega and Fomalhaut, the $K$-band magnitude difference for photospheres between Fomalhaut and Sirius is $2.35 \mathrm{mag}$, and $1.39 \mathrm{mag}$ between Vega and Sirius. For measurements in the IRAC bands, we adopted the results from Marengo et al. (2009) who used the PSF fitting technique to recover accurate photometry for saturated sources. The magnitude differences between Fomalhaut and Sirius are $2.36 \mathrm{mag}, 2.36 \mathrm{mag}$, $2.36 \mathrm{mag}$, and $2.33 \mathrm{mag}$ at $3.6,4.5,5.8$, and $8 \mu \mathrm{m}$, respectively (to be discussed further by Espinoza et al. in preparation). The magnitude differences between Vega and Sirius are $1.38 \mathrm{mag}$, $1.38 \mathrm{mag}, 1.38 \mathrm{mag}$, and $1.35 \mathrm{mag}$ at 3.6, 4.5, 5.8, and $8 \mu \mathrm{m}$, respectively. Furthermore, Midcourse Space Experiment (MSX) also measured both Vega and Sirius. The measured $8 \mu \mathrm{m}$ flux of Vega is lower by $1 \%$ compared to the $M S X$ predicted flux based on the measurement of Sirius (Price et al. 2004). We adopt a magnitude difference of $1.36 \mathrm{mag}$ at $8 \mu \mathrm{m}$ between Vega and Sirius.

With the nominal uncertainty for these measurements (0.01 mag), there is no evidence for excesses from 2.2 to $8 \mu \mathrm{m}$ (after excluding the $K$-band excesses from interferometric measurements) for both systems. We used appropriate Kurucz atmospheric models for photospheric predictions in other wavelengths by normalizing the model fluxes from 2.2 to $8 \mu \mathrm{m}$ to the measured photometry that has been transferred to the absolute calibration scale proposed by Rieke et al. (2008). The parameters in the Kurucz model are $T_{\text {eff }}=9500 \mathrm{~K}$ and $\log g=4.0$ for Vega, and $T_{\text {eff }}=8750 \mathrm{~K}$ and $\log g=4.0$ for Fomalhaut. Given the accurate Hipparcos parallax measurements (van Leeuwen 2007), the integrated luminosity is $17.45 L_{\odot}$ for Fomalhaut and $56.05 L_{\odot}$ for Vega (viewing from pole-on).

Using these normalized Kurucz models, we can then predict the photospheric level at the wavelengths of interest and determine the excess near the stars by subtracting off the stellar contribution from the IRS spectra presented in Section 2.1. Note that the Kurucz models in the infrared wavelengths are basically in the Rayleigh-Jeans regime.

\subsection{PACS Measurements for the Vega Central Source}

The Vega system is viewed pole-on, and its disk has previously been resolved at various wavelengths (Holland et al. 1998; Heinrichsen et al. 1998; Su et al. 2005; Marsh et al. 2006; Sibthorpe et al. 2010). As shown in Figure 3, the PACS $70 \mu \mathrm{m}$ image appears to be centrally peaked with a smoothed, axis-symmetric extended halo. The size of the Vega cold disk observed in the higher resolution Herschel data (Sibthorpe et al. 2010) agrees with the one observed in the Spitzer data ( $\mathrm{Su}$ et al. 2005). The stellar photosphere is about $0.81 \mathrm{Jy}$ at $70 \mu \mathrm{m}$, consistent with the centrally peaked morphology seen at that wavelength. The FWHM of the central source is $5^{\prime \prime} .6 \times 5^{\prime \prime} .3$ measured on a field of $21^{\prime \prime} \times 21^{\prime \prime}$ centered on the star, which is slightly more extended than the measured FWHM of the PSF star, $\alpha$ Boo $\left(5^{\prime \prime} .5 \times 5^{\prime \prime} .2\right)$. To minimize the influence of the cold ring (peaked at radius of $\sim 11^{\prime \prime}-14^{\prime \prime}$, see Figure 4 ) in estimating the photometry of the central source, small aperture sizes with appropriate aperture corrections should be used. On the other hand, the aperture has to be large enough to contain most of the flux and to minimize the centroid uncertainty. We tried several aperture settings from $3^{\prime \prime}$ to $5^{\prime \prime} .5$ (FWHM) with and without a sky annulus and used the PSF star $\alpha$ Boo to derive the values of aperture correction. The resultant fluxes range from $1.00 \mathrm{Jy}$ to $1.62 \mathrm{Jy}$ with a median value of $1.01 \mathrm{Jy}$ (using an aperture of $5^{\prime \prime}$ and sky annulus between $5^{\prime \prime}$ and $\left.7^{\prime \prime}\right)$. At $160 \mu \mathrm{m}$, the beam $(\mathrm{FWHM})$ is $11^{\prime \prime} .6 \times 10^{\prime} .1$ (measured from $\alpha$ Boo), making it very

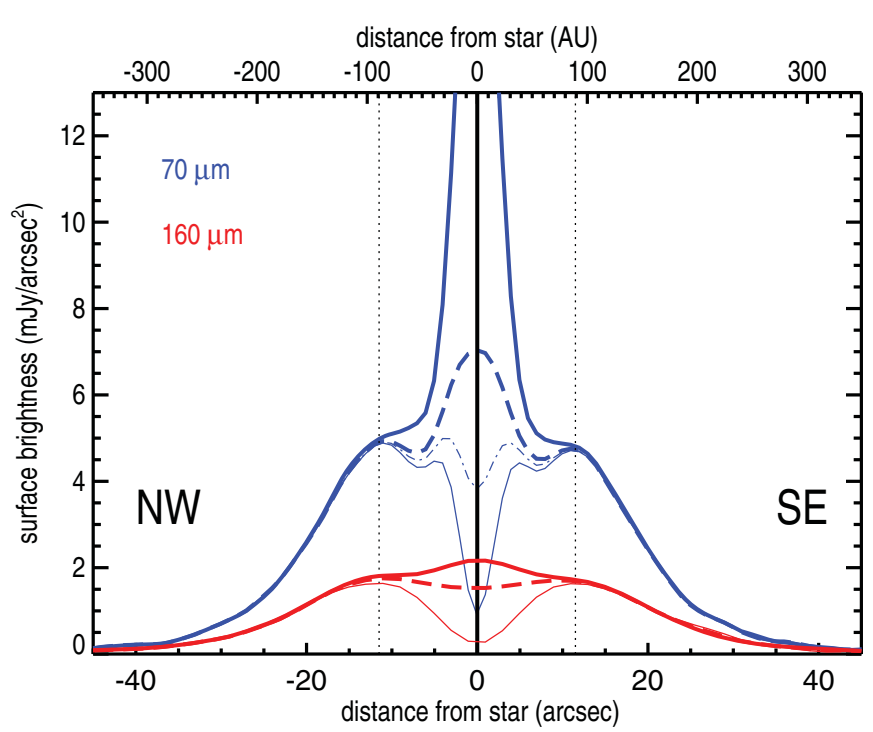

Figure 4. Surface brightness cuts for the Vega system. These cuts were centered at the star position and represent the mean value over a width of 5 pixels along the PA of $120^{\circ}$ (along the horizontal axis in this image). The profiles at $70 \mu \mathrm{m}$ are shown in blue color while the ones at $160 \mu \mathrm{m}$ in red. The original cuts (without PSF subtraction) are shown in thick solid lines, and the long-dashed lines represent the cuts after the subtraction of photosphere. Other thin lines represent the cuts made with different levels of subtractions (scaling of the PSF). For details see Section 3.2.

(A color version of this figure is available in the online journal.)

difficult to estimate the photometry of the central source alone without significant contamination from the flux of the cold ring. A $5^{\prime \prime}$ aperture without sky annulus gives a total flux of $0.56 \mathrm{Jy}$, which should be considered as an upper limit since it contains some fraction of the cold ring contribution. The photosphere of Vega is $0.15 \mathrm{Jy}$ at $160 \mu \mathrm{m}$. Therefore, the excess flux at the star position, based on small aperture photometry, is $0.2 \mathrm{Jy}$ at $70 \mu \mathrm{m}$ and $<0.4 \mathrm{Jy}$ at $160 \mu \mathrm{m}$.

To illustrate our PSF-subtraction approach, cuts were made along a PA of $120^{\circ}$ (corresponding to the horizontal axis of the displayed image) of the Vega system, with the NW side on the negative side of the $x$-axis. These cuts, shown in Figure 4, were centered at the stellar position at $70 \mu \mathrm{m}$ and represented the mean value over a width of 5 pixels (i.e., $5^{\prime \prime}$ and $10^{\prime \prime}$ at 70 and $160 \mu \mathrm{m}$, respectively). No significant asymmetry in disk brightness nor center offset were seen in these cuts. After subtracting a photospheric scaled $\alpha$ Boo PSF, an additional point-like source is clearly present in the resultant cut (blue dashed line in Figure 4) at $70 \mu \mathrm{m}$. The maximum of the pointsource contribution (star and unresolved disk) can be estimated by forcing the peak values (single pixel) matched between the Vega and $\alpha$ Boo data. A flux density of $1.19 \mathrm{Jy}$ for such a PSF subtraction is required and its resultant profile is shown as the thin solid blue line in Figure 4, suggesting a maximum brightness of $<0.38 \mathrm{Jy}$ for this unresolved disk at $70 \mu \mathrm{m}$. Using the flux obtained with the small aperture photometry $(0.2 \mathrm{Jy}$ for the unresolved disk), the resultant profile is shown as the blue dotted-dashed line in Figure 4, relatively flat in the central $\pm 5^{\prime \prime}$ region as expected. Therefore, the brightness of the unresolved disk in the Vega system is $\sim 0.2$ Jy with a maximum value $<0.38$ at $70 \mu \mathrm{m}$. Due to the uncertainty of the PSF (as much as $10 \%$ at $70 \mu \mathrm{m}$; Kennedy et al. 2012) and that of flux calibration, we simply assume a conservative lower-bound error of $20 \%$ (i.e., a minimum flux of $0.16 \mathrm{Jy}$ at $70 \mu \mathrm{m}$ for the central unresolved disk). At $160 \mu \mathrm{m}$, the maximum scaled PSF is $\sim 0.45 \mathrm{Jy}$ by 


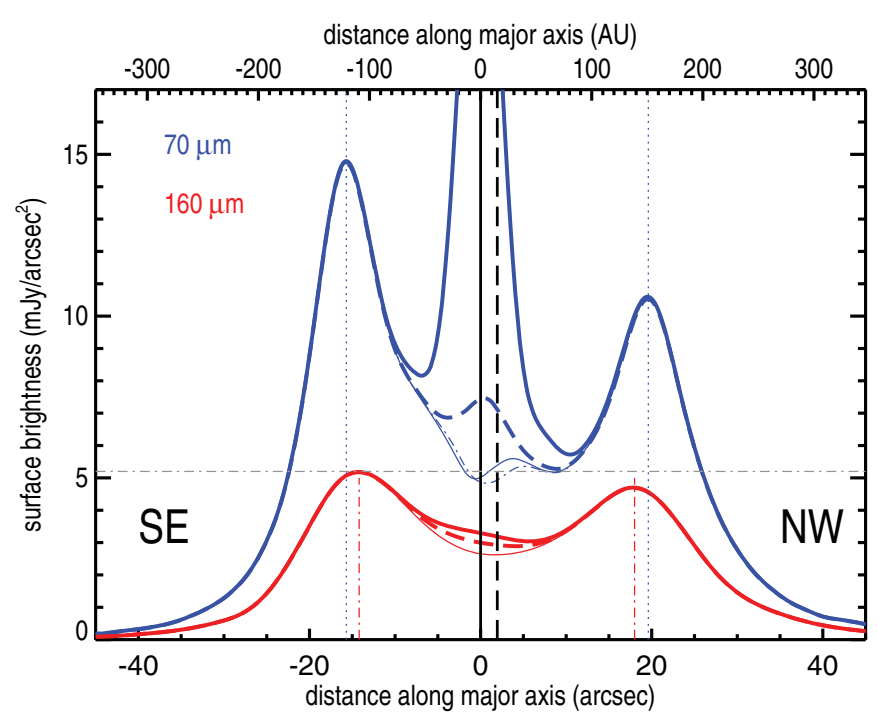

Figure 5. Surface brightness cuts along the major axis of the Fomalhaut system. These cuts were centered at the star position (marked as the solid vertical line) and represent the mean value over a width of four pixels along the minor axis. Line styles and colors are similar to those used in Figure 4. The dotted and dotted-dash vertical lines mark the peak positions of the ansae at 70 and $160 \mu \mathrm{m}$, respectively, while the long-dashed vertical line represents the mid-point of the cold ring.

(A color version of this figure is available in the online journal.)

forcing the peak value to zero, suggesting a maximum brightness of $<0.3 \mathrm{Jy}$ for this unresolved disk component.

\subsection{PACS Measurements for the Fomalhaut Central Source}

Since the Fomalhaut debris system is inclined by $67^{\circ}$, it is very difficult to separate the point source from the cold ring, even using very small apertures. One can still estimate the maximum brightness of the unresolved component by assuming that there is no contamination from other sources inside a very small aperture. For an aperture radius of $3^{\prime \prime}$, the encircled flux is $0.95 \mathrm{Jy}$ at $70 \mu \mathrm{m}$ and $0.77 \mathrm{Jy}$ at $160 \mu \mathrm{m}$ after applying appropriate aperture corrections. Taking out the contribution of the stellar photosphere $(0.368 \mathrm{Jy}$ and $0.07 \mathrm{Jy}$ at 70 and $160 \mu \mathrm{m}$, respectively, based on our stellar photospheric model), the unresolved disk component has a maximum flux of $<0.58 \mathrm{Jy}$ and $<0.7 \mathrm{Jy}$ at 70 and $160 \mu \mathrm{m}$, respectively.

Similar to Vega, cuts were made along the major-axis of the disk and shown in Figure 5. These cuts were centered at the stellar position at $70 \mu \mathrm{m}$ and represented the mean value over a width of 4 pixels (i.e., $4^{\prime \prime}$ and $8^{\prime \prime}$ at 70 and $160 \mu \mathrm{m}$, respectively). The center of the outer cold ring (the dashed, vertical line in Figure 5) was estimated by the mid-point of the two bright peaks (marked as dotted, vertical lines for $70 \mu \mathrm{m}$ and dot-dashed, vertical lines for $160 \mu \mathrm{m}$ in Figure 5) at both wavelengths. The $160 \mu \mathrm{m}$ ansae peak closer to the ring center than the ones at $70 \mu \mathrm{m}$, which was first reported by Acke et al. (2012) and suggested to be due to blurring in the large PSF at $160 \mu \mathrm{m}$. Although the peak positions at 70 and $160 \mu \mathrm{m}$ are not the same, the cold ring centers at the same position at both 70 and $160 \mu \mathrm{m}$. We used a PSF star, $\alpha$ Tau, for photospheric subtraction by scaling it to match the expected photospheres $(0.368 \mathrm{Jy}$ and $0.07 \mathrm{Jy}$ at 70 and $160 \mu \mathrm{m})$. The photospheresubtracted cuts are shown in long-dashed lines in Figure 5. At $70 \mu \mathrm{m}$, it is clear that an additional source of emission is present at the center of the disk. We tried two different PSF subtractions to estimate the brightness of this additional component. First, we arbitrarily increased the scaling of $\alpha$ Tau, fixed in the stellar photosphere position, until the central region (from $-5^{\prime \prime}$ to $+5^{\prime \prime}$ ) has a relatively flat distribution in the cut. The resultant cut is shown as a thin solid blue line with an additional scale of $0.17 \mathrm{Jy}$. Second, we subtract a second $\alpha$ Tau PSF by adjusting both the position and the scaling until the resultant cut is relatively flat (dot-dashed line in Figure 5). The second method gives a scale of $0.165 \mathrm{Jy}$ for this additional source. Combining both methods, we conclude that the central unresolved component is $0.17 \mathrm{Jy}$ at $70 \mu \mathrm{m}$. There is no easy way to estimate the error associated with this number since it depends on the detailed structures of various components; we simply assume a $20 \%$ error for further analysis. At $160 \mu \mathrm{m}$, it is not obvious that an additional source is required in the system. We estimated the upper limit of such a component by increasing the scaling factor of $\alpha$ Tau fixed at the star position (any potential offset between the star and this inner component is washed out by the large beam size at $160 \mu \mathrm{m})$. An upper limit of $0.08 \mathrm{Jy}$ at $160 \mu \mathrm{m}$ was inferred.

In summary, the central unresolved disk component in the Fomalhaut system is about $0.17 \mathrm{Jy}$ at $70 \mu \mathrm{m}$, with upper- and lower-bound fluxes of $0.58 \mathrm{Jy}$ and $0.136 \mathrm{Jy}$ (assuming 20\% uncertainty), and $<0.08 \mathrm{Jy}$ at $160 \mu \mathrm{m}$, based on the analysis of the data only (without invoking any assumption of modeling). Our values are consistent with the best-fit model presented in Acke et al. (2012). Their model estimates that the fluxes of the central point source (star + unresolved disk component) are $0.54 \mathrm{Jy}$ ( $5 \%$ of the total flux) and $0.124 \mathrm{Jy}$ ( $2 \%$ of the total flux) at 70 and $160 \mu \mathrm{m}$, respectively, implying that the unresolved disk accounts for flux of $0.172 \mathrm{Jy}$ at $70 \mu \mathrm{m}$ and $0.054 \mathrm{Jy}$ at $160 \mu \mathrm{m}$.

\section{ANALYSIS}

\subsection{Unresolved Warm Excess in the Vega System}

The Vega Spitzer $24 \mu \mathrm{m}$ observation ( $\mathrm{Su}$ et al. 2005) was severely saturated, making it difficult to constrain the brightness of this unresolved component without invoking some modeling assumptions. Therefore, we seek other relevant measurements in the mid-infrared to validate the excess levels seen in the IRS spectrum. Tokunaga (1984) measured a handful of nearby A-type stars at $20 \mu \mathrm{m}$ using the IRTF bolometer with a beam size of $5^{\prime \prime}$ to define the $20 \mu \mathrm{m}$ magnitude system (relative to Vega). Only two stars, $\alpha$ CMa (Sirius) and $\gamma \mathrm{UMa}$, in his list do not have a $24 \mu \mathrm{m}$ excess. We used the color $V-[20]$ of these two stars to extract the excess of Vega at $20 \mu \mathrm{m}$. We adopt $V$ of -1.40 and $2.40 \mathrm{mag}$ for $\alpha \mathrm{CMa}$ and $\gamma \mathrm{UMa}$, suggesting a photosphere color $V-[20]$ of -0.04 mag in Tokunaga's system. Based on the observed $V-[20]$ color of 0.03 , the excess of Vega is $\sim 7 \%$ above the photosphere (i.e., $0.7 \mathrm{Jy}$ ). It is difficult to assess the errors associated with the $20 \mu \mathrm{m}$ measurement; therefore, we simply assume a maximum $50 \%$ error at this wavelength. Another source of mid-IR measurements for Vega comes from $M S X$ photometry where the excesses (relative to Sirius) at 14.65 and $21.34 \mu \mathrm{m}$ have been reported by Price et al. (2004) to be $4 \%$ and $17 \%$ above the photosphere (i.e., excesses of $0.7 \mathrm{Jy}$ at $14.65 \mu \mathrm{m}$ and $1.5 \mathrm{Jy}$ at $21.34 \mu \mathrm{m}$ ), respectively. The contamination from the cold ring needs to be taken into account given the large beam size of $M S X$ (a resolution of $\sim 20^{\prime \prime}$ ). Assuming a typical dust temperature of $60 \mathrm{~K}$ for the cold ring and normalizing the cold ring flux observed in the far-infrared (Su et al. 2005), the flux contamination from the cold ring is less than $0.1 \%$ of the photosphere at $14.65 \mu \mathrm{m}$, and $\sim 4 \%$ of the photosphere at $21.34 \mu \mathrm{m}$. The nominal error for the $M S X$ 


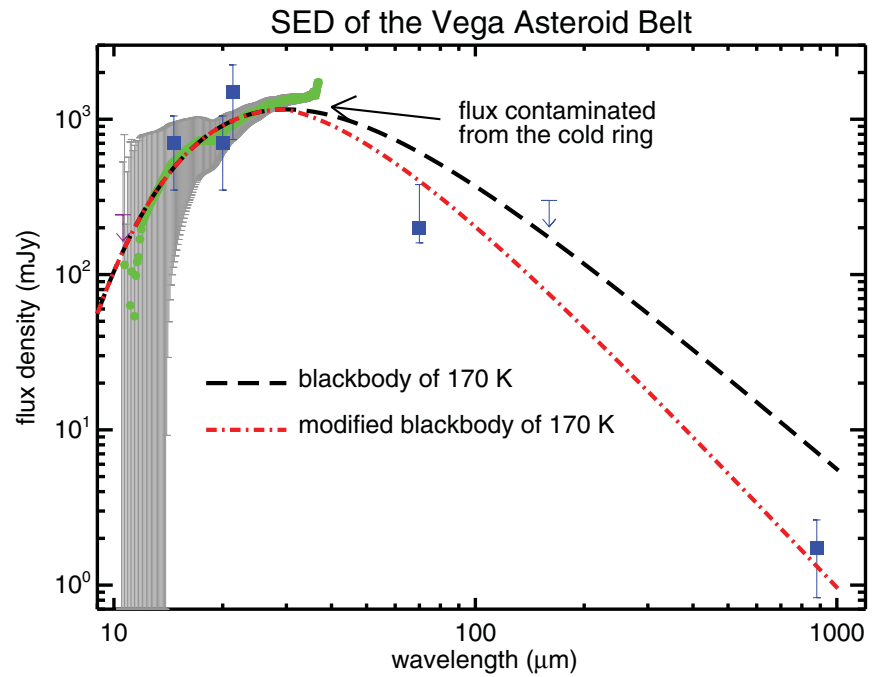

Figure 6. Excess SED of the inner warm component in Vega. The photospheric subtracted IRS spectrum is shown as green dots with errors (including the $2 \%$ errors from the photosphere) shown as gray area; other IR and submillimeter excess photometry are shown as blue squares. For comparison, blackbody curves of $170 \mathrm{~K}$ are also shown with $B_{\lambda}$ as the long-dashed line and $\lambda^{-0.5} B_{\lambda}$ as the dotted-dash line. The $10.6 \mu \mathrm{m}$ BLINC/MMT $1 \sigma$ upper limit is also shown.

(A color version of this figure is available in the online journal.)

measurements is $\sim 1.5 \%$ (Rieke et al. 2008), which includes the errors from stellar photospheric prediction. Including the possible contamination from the cold ring, the final errors are $0.31 \mathrm{Jy}$ and $0.38 \mathrm{Jy}$ at $1 \sigma$ for the excesses at 14.65 and $21.34 \mu \mathrm{m}$. The Vega system was recently observed by the Submillimeter Array (SMA) at $880 \mu \mathrm{m}$ (Hughes et al. 2012). Interestingly, the spatially unresolved $880 \mu \mathrm{m}$ flux within $5^{\prime \prime}$ of the Vega photosphere lies slightly above the predicted photospheric value (see Figure 6), although only at the less than $2 \sigma$ level. Combined with our estimates from the PACS images and the IRS spectrum, the excess SED of the central component in the Vega system is shown in Figure 6. We note that Liu et al. (2004) also report an upper limit ( $\sim 250 \mathrm{mJy})$ of $0.7 \%(1 \sigma)$ of the photospheric level at $10.6 \mu \mathrm{m}$, using nulling interferometry (BLINC/MMT) that probes a region less than $1^{\prime \prime} .5(12 \mathrm{AU})$ from the star.

The spectral shape $(\lambda<30 \mu \mathrm{m})$ of the inner component is well represented by a blackbody of $170 \mathrm{~K}$, just above the temperature at which water ice sublimates in vacuum. This temperature corresponds to a distance $(\sim 2.7 \mathrm{AU})$ in the middle of our asteroid belt in the solar system, but $\sim 14 \mathrm{AU}$ in the Vega system (using an average stellar luminosity of $37 L_{\odot}$ viewed by the dust along the equator of the star; Aufdenberg et al. 2006) assuming blackbody-like emitters.

\subsection{Unresolved Warm Excess in the Fomalhaut System}

The existence of a close (unresolved) warm component around the Fomalhaut disk was first suggested by the Spitzer $24 \mu \mathrm{m}$ observation where an additional point-like source $(0.6 \pm$ $0.2 \mathrm{Jy}$ ) along with the expected stellar photosphere is required to fit the resolved disk image at $24 \mu \mathrm{m}$ (Stapelfeldt et al. 2004). The central component of the Fomalhaut system was also tentatively detected by the ALMA observation at $850 \mu \mathrm{m}$ (Boley et al. 2012). A flux density of $\sim 4.4 \mathrm{mJy}$ at the star position was estimated after the primary beam correction, which is quite uncertain at the star position because the ALMA observation was centered at Fomalhaut b. The expected photosphere at $850 \mu \mathrm{m}$ is $\sim 2 \mathrm{mJy}$ (not $3 \mathrm{mJy}$ as stated in Boley et al. 2012). Therefore, the

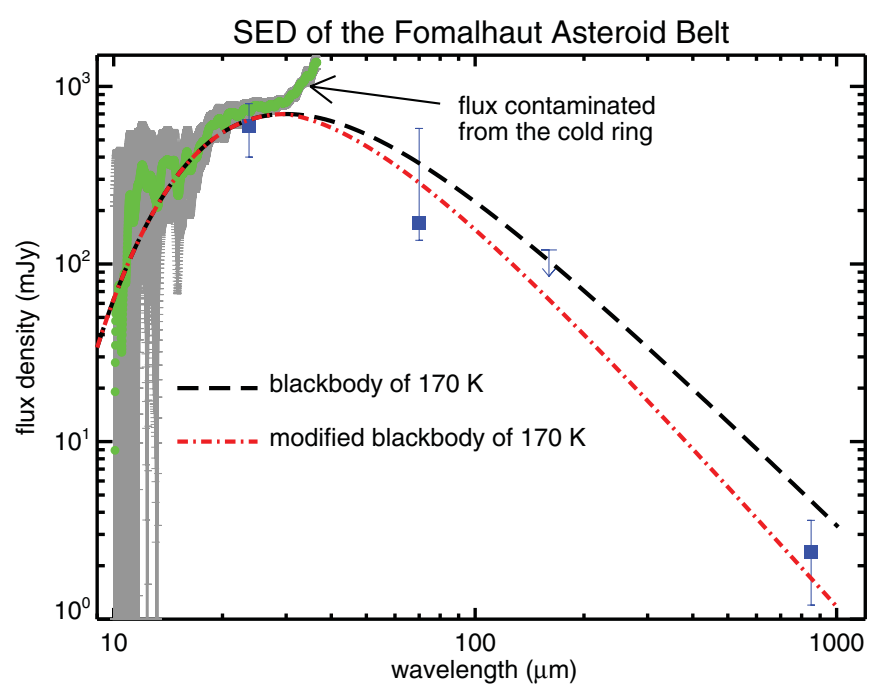

Figure 7. Excess SED of the inner warm component in Fomalhaut. Symbols and lines are similar to what were used in Figure 6. The upper error bar at $70 \mu \mathrm{m}$ represents the (unlikely) maximum value estimated from small aperture photometry and the lower error bar is $20 \%$ lower than the estimate from PSF subtraction. Other error bars are shown as $1 \sigma$. For comparison, blackbody curves of $170 \mathrm{~K}$ are also shown with $B_{\lambda}$ as the long-dashed line and $\lambda^{-0.3} B_{\lambda}$ as the dotted-dash line.

(A color version of this figure is available in the online journal.)

excess at $850 \mu \mathrm{m}$ could be as high as $2.4 \mathrm{mJy}$. We simply took this at face value and assumed a $50 \%$ error (as maximal). The excess SED for the Fomalhaut central unresolved component is shown in Figure 7 along with the excess spectrum measured by IRS.

We note that Acke et al. (2012) suggested ionized gas (free-free emission from a hot stellar wind) as being responsible for the unresolved central excess from $K$ band to $70 \mu \mathrm{m}$, and derived $F_{v} \propto v^{0.8 \pm 0.1}$ power law. With their derived power law, the excess at $850 \mu \mathrm{m}(350 \mathrm{GHz})$ is expected to be $18-23 \mathrm{mJy}$, which is clearly not consistent with the ALMA observation. In addition, this power-law spectrum is inconsistent with the 2.2-5.8 $\mu \mathrm{m}$ colors discussed in Section 3.1. Instead, we suggest that the excess emission detected longward of $\sim 13 \mu \mathrm{m}$ arises from thermal dust emission. Similar to the warm component in the Vega system, the spectral shape $(\lambda<30 \mu \mathrm{m})$ of the inner component in Fomalhaut is also well represented by a blackbody of $170 \mathrm{~K}$, suggesting a radial distance of $\sim 11$ AU from the star for blackbody-like grains.

\subsection{Asteroid-belt Analogs}

The warm excesses detected in both systems are restricted to the vicinity of the star, i.e., unresolved at various wavelengths. The amount of warm excess emission is derived either from resolved images where the central component is (mostly) separated from the cold ring, or from the spectra taken centered at the star position. Given the sizes of the IRS slits, some amount of the flux from the cold rings is expected to fall into the slit, especially at wavelengths longer than $\sim 30 \mu \mathrm{m}$. It is difficult to estimate the exact flux contamination without further modeling because it depends on the geometry of the cold ring at different wavelengths. One can estimate the maximum flux contamination in the worst scenario case by assuming that the warm and cold components are spatially coincident. Therefore, the maximum flux contamination is $\lesssim 1 \%$ of the photosphere for $\lambda<20 \mu \mathrm{m}$ and could be up to $50 \%$ at $33 \mu \mathrm{m}$ given the 
typical dust temperature of the cold ring $(\sim 70-50 \mathrm{~K})$. Thus, the derived dust temperature ( $\sim 170 \mathrm{~K}$ in both systems) based on the data shortward of $30 \mu \mathrm{m}$ has very little contamination from the cold ring. In other words, the excess arises from material less than $3^{\prime \prime}$ (unresolved with an FWHM $\sim 6^{\prime \prime}$ ) from the star in both cases. Similar to the definition of habitable zones around stars, debris disk structures should be identified and characterized in terms of dust temperatures rather than physical distances so that the heating power of different spectral types of stars is taken into account and common features in disks can be discussed and compared directly. The characteristic temperature of $\sim 170 \mathrm{~K}$ for the excesses suggests that they are asteroid-belt analogs. It corresponds to a distance of $2^{\prime \prime}$ ( $\sim 14$ AU) from Vega (using the lower luminosity viewed by the dust in the equatorial direction) or of $1^{\prime \prime} .5(\sim 11 \mathrm{AU})$ from Fomalhaut assuming blackbody radiators. The resultant location of the asteroid belt given a dust temperature depends greatly on grain properties. Using astronomical silicates (Laor \& Draine 1993), the dust can be as close as 1".3 (10 AU) from Vega, and $1^{\prime \prime}$ (8 AU) from Fomalhaut using grains with a radius of $10 \mu \mathrm{m}$. Furthermore, the IRAM PdBI observations at $1.3 \mathrm{~mm}$ by Piétu et al. (2011) do not find any excess within 2".5 while the SMA data by Hughes et al. (2012) suggest a tentative excess within $5^{\prime \prime}$ from the star, suggesting that the warm excess around Vega likely arises from emission outside a radius of 1".3 (10 AU) from the star. The fractional luminosity is $7 \times 10^{-6}$ for the Vega warm component and $2 \times 10^{-5}$ for the Fomalhaut warm component, much more luminous than our current zodiacal cloud $\left(10^{-8}\right.$ to $10^{-7}$; Dermott et al. 2002).

The origin of the warm excess in the vicinity of the star is unknown. One hypothesis is that the warm excess originates from dust grains in the cold belt, which are transported inward by P-R and/or stellar wind drag, as suggested by Reidemeister et al. (2011) for the outer warm belt of the $\epsilon$ Eri debris system. In the Fomalhaut system, the presence of an inner disk, extending inward up to $~ 35 \mathrm{AU}(4 . .5)$ and composed of grains draggedin from the cold planetesimal belt, has been suggested by Acke et al. (2012) to explain the PACS $70 \mu \mathrm{m}$ profile. Conventionally, a P-R-transported flow has a constant surface density and is expected to extend to the star, resulting in a surface brightness profile that is centrally peaked at the star. However, Acke et al. (2012) find that the inner disk in Fomalhaut has a clear truncation (outside the unresolved warm component). Therefore, neither the warm nor the hot excess observed in the Fomalhaut system is unlikely to have arisen from the inward transport of grains by $\mathrm{P}-\mathrm{R}$ drag. On the other hand, we cannot rule out such a possibility (either solely or partially due to the dragged-in grains) in the Vega system (it is centrally peaked at $70 \mu \mathrm{m}$ ) without detailed modeling for the whole system at multiple wavelengths (K. Y. L. Su et al. in preparation). Nevertheless, the observed dust temperature $(170 \mathrm{~K})$ of the warm excess suggests that this component does not extend all the way to the star; instead, an inner truncation $\left(>1^{\prime \prime}\right)$ is required.

Furthermore, the tentative detection of the central component at submillimeter wavelengths in both systems argues for the presence of large grains, suggesting that the warm emission likely arises from a full spectrum of particle sizes similar to the cold planetesimal belt, i.e., a form of planetesimal belt like our asteroid belt. The wavelength-dependent power index at long wavelengths $\left(F_{v} \sim \lambda^{-l}\right)$ is a measure of the grain size distribution in a collision-dominated debris disk (Wyatt \& Dent 2002; Gáspár et al. 2012). The cold planetesimal belts observed in bright debris disks have much steeper slopes compared to Rayleigh-Jeans slope (e.g., Gáspár et al. 2012). Similar behavior is seen in the warm component in the Vega and Fomalhaut systems (see Figures 6 and 7), implying the presence of large grains. Limited by the uncertainties in the current observations, the exact slope of the warm component at long wavelengths cannot be determined accurately. Future highresolution observations in the submillimeter and millimeter will help shed light on the nature of the warm excesses.

One interesting note on the nature of these warm excesses is that the observed levels of dust $\left(f_{d} \sim 7 \times 10^{-6}\right.$ for Vega and $f_{d} \sim 2 \times 10^{-5}$ for Fomalhaut) are consistent with being the in situ, steady-state collisional evolution of large parent bodies for the lifetime of the stars ( $400 \mathrm{Myr})$. The maximum fraction luminosity $\left(f_{\max }\right)$ for such a system can be estimated using Equation (18) from Wyatt et al. (2007), where $f_{\max }$ is on the order of (2-3) $\times 10^{-5}$ for both systems, assuming an asteroid belt at $\sim 10 \mathrm{AU}$ with a width of $\sim 1 \mathrm{AU}$ and consisting of $\sim 10 \mathrm{~km}$ (diameter) planetesimals in collisional cascades. Due to the uncertainties of some parameters in the model, Wyatt et al. (2007) suggest that a transient event producing the observed dust is only required when $f_{\text {obs }} \gg 10^{3} f_{\max }$. Thus, the observed dust in the warm component is consistent with it being generated through collisional grinding in an asteroid belt in both systems.

\section{DISCUSSION}

Vega and Fomalhaut really live up to their names as debris disk twins: both possess a hot $2 \mu \mathrm{m}$ excess revealed through interferometric observations, a cold $(\sim 50 \mathrm{~K})$ belt analogous to our Kuiper belt that has been in the spotlight of space infrared facilities, and a warm $(\sim 170 \mathrm{~K})$ belt analogous to our asteroid belt. This warm-and-cold-belt debris architecture has also been identified in HR 8799 (Su et al. 2009) and $\epsilon$ Eri (Backman et al. 2009). In Section 5.1, we first discuss the possible origin of these two-belt systems in terms of their formation and evolution. Taken together with the known properties of planets and mass limits from non-detection, we speculate that the large gap between the two belts is likely to be sustained by multiple (lowmass) planets. We then review the properties of $2 \mu \mathrm{m}$ excess in Section 5.2 and suggest an alternative explanation for the hot dust component.

\subsection{Origin of the Two-belt Systems}

In our solar system, the locations of the minor bodies that failed to form planets are elegantly arranged and sculpted by planetary perturbations over the course of $4.5 \mathrm{Gyr}$ evolution. The inner edge of the Kuiper belt's dusty disk is thought to be maintained by massive planets (Liou \& Zook 1999), whereas the more tenuous asteroid-belt dust (i.e., zodiacal cloud) has a structure also determined by gravitational perturbations via both the giant and terrestrial planets (Dermott et al. 1994; Murray et al. 1998). It has been suggested that the dominant source of dusty debris inside $\sim 5 \mathrm{AU}$ in our solar system results from the breakup of asteroids (e.g., Dermott et al. 2002). However, a recent dynamical model incorporating multiple sources (asteroids and short- and long-period comets) by Nesvorný et al. (2010) suggests that particles originating from Jupiter-family (short-period) comets dominate the mid-infrared emission in the zodiacal cloud while the contribution of asteroidal dust is $<10 \%$. The origin of our own zodiacal cloud is still a matter of considerable debate, making an understanding of the warm dust around nearby stars (i.e., exo-zodi) even more valuable. 


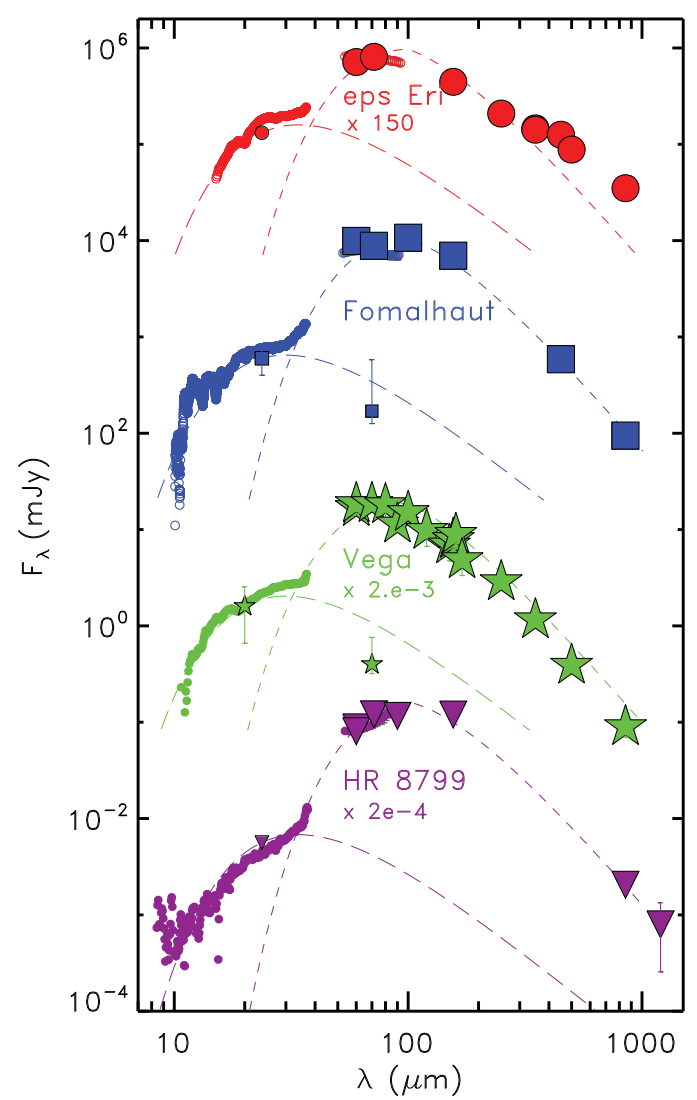

Figure 8. Excess SEDs for $\epsilon$ Eri, Fomalhaut, Vega, and HR 8799 where measurements are shown in various symbols with colors differentiating the objects, and large size symbols being the integrated photometry while smaller ones representing spectroscopic measurements. The photometry of the warm components is also shown as the smaller symbol. The excess of each system is well represented by two (warm as long-dashed line and cold as dashed line) blackbody emissions: $\epsilon$ Eri: $150 \mathrm{~K}$ and $50 \mathrm{~K}$, Fomalhaut: $170 \mathrm{~K}$ and $50 \mathrm{~K}$, Vega: $170 \mathrm{~K}$ and $50 \mathrm{~K}$, and HR 8799: $150 \mathrm{~K}$ and $45 \mathrm{~K}$. Note that the cold component is modeled with modified blackbody as $\lambda^{-0.9} B_{\lambda}$ in order to fit the submillimeter observations.

(A color version of this figure is available in the online journal.)

Dust locations solely based on temperatures derived from ex-solar debris disk SEDs are ambiguous. However, based on resolved images at multiple wavelengths, four systems-Vega, Fomalhaut, $\epsilon$ Eri, ${ }^{12}$ and HR 8799-clearly have two dust belts. The disk SEDs of these four systems are shown together in Figure 8 for easy comparison. As discussed in Section 4.3, the dust observed in the warm components for both Vega and Fomalhaut is consistent with being generated in a steady-state collisional cascade in a planetesimal belt. A similar conclusion is obtained for the other two systems. For HR 8799, the warm dust component has a temperature $\sim 150 \mathrm{~K}$ and fractional luminosity $f_{d} \sim 2 \times 10^{-5}$ (Su et al. 2009); the latter is much less than the maximum fractional luminosity for steady state collisional dust production, $f_{\max } \approx 3 \times 10^{-4}$, estimated for a belt at 8 AU at HR 8799's age of $\sim 30$ Myr. For the $\epsilon$ Eri system, the warm component has $f_{d} \sim 3 \times 10^{-5}$ and a temperature of $\sim 150 \mathrm{~K}$ (Backman et al. 2009); the latter is compatible with $f_{\max } \approx 8 \times 10^{-6}$ for a belt at $3 \mathrm{AU}$ at an age of $\sim 800 \mathrm{Myr}$. Based on the similarity of the observed SEDs and resolved disk structures, it is very likely that the warm components in these four systems originate in well-separated planetesimal belts

\footnotetext{
12 For the $\epsilon$ Eri disk, we refer to the inner warm belt that dominates the emission at $24 \mu \mathrm{m}$ rather than the outer warm belt that emits prominently at $55 \mu \mathrm{m}$ (Backman et al. 2009).
}

(remnants of planet formation) resembling the configuration of our own solar system, which has two left-over planetesimal belts: an asteroid belt near the water-frost line, between Mars and Jupiter, with a characteristic temperature of $\sim 170-150 \mathrm{~K}$, and a Kuiper belt at $\sim 30-55$ AU with dust emission peaked at $\sim 50 \mathrm{~K}$

There are two aspects of the two-belt systems that must be explained. The first one is related to how these two separate belts were created, and the other one addresses how the system maintains such a large gap without dust filling it in. Mechanisms to explain both the formation and evolution of the two belt systems are required to fully account for the observed pattern. Planetesimal belts can be expected in regions where there was not enough material or not enough time to form a planet, because once formed, a planet would scatter or accrete the surrounding planetesimals. Therefore, the stable locations of left-over planetesimals are governed by where the giant planets form and their migration history, which may include strong dynamical instabilities. Consequently, the location of the observed dust is not expected to show much order among systems since it greatly depends on the numbers and positions of the giant planets and their migration history. In addition, it is an observational fact that higher mass stars harbor a more massive protoplanetary disk (Natta et al. 2000; Williams \& Cieza 2011) and more giant planets (Johnson et al. 2010). Generally, one does not expect to find similarity in planetary configurations between low-mass (i.e., low luminosity) and high-mass (i.e., high luminosity) stars.

Among the four systems shown in Figure 8, only $\epsilon$ Eri is a solar-like star and the rest are early-type stars. The luminosity (the dust heating power) difference ranges roughly two orders of magnitude among them, and yet the characteristic dust temperatures of the warm and cold belts are very similar. It is interesting to note that many unresolved systems also have a similar two-belt configuration in the dust distribution based on their temperatures derived from unresolved excess emission (Chen et al. 2009; Morales et al. 2011). The fact that the temperatures of the warm belts peak at similar temperatures $(\sim 170-190 \mathrm{~K})$ between early-type and solar-like systems (Morales et al. 2011), however, suggests that temperature-sensitive mechanisms play a major role in determining planetesimal configuration in the warm belts. Giant planets are expected to form right outside the water-frost line where the amount of solid material and dynamical timescales favor the formation process (Kretke \& Lin 2007).

The young HR 8799 system has four giant planets (Marois et al. 2008, 2010) separating the inner and outer dust belts ( $\mathrm{Su}$ et al. 2009). These four planets are very massive $\left(\sim 7-10 M_{J}\right)$, so that they dominate the dynamics in the HR 8799 system. Dust particles spiraling inward from the cold belt under P-R drag are likely to be dynamically scattered and ejected by one of the planets before they reach the inner region. Thus, the warm component in the HR 8799 system is unlikely to arise from grains generated in the cold planetesimal belt. Comets on plunging orbits originating from the cold belt could possibly cross over and then break-up or sublimate to deliver dust interior to the giant planets. However, it is difficult to estimate the inward flux of cometary bodies inside the orbits of the giant planets without detailed numerical simulations such as the one done by Bonsor et al. (2012). Furthermore, while the comet delivery scenario proposed by Nesvorný et al. (2010) might work for our solar system at its current age, it is not clear that this scenario is directly applicable for younger systems (such as the ones we 
discussed here) where in situ dust generation in the younger, more massive planetesimal belts is expected to dominate.

Of the remaining three systems, Fomalhaut is the only other one that harbors a directly detected planet, Fomalhaut b (Kalas et al. 2008). Although the reality of Fomalhaut b has been questioned because the spectrophotometry of Fomalhaut $b$ cannot be reconciled with models for thermal emission from a young giant planet, a problem originally acknowledged in Kalas et al. (2008) and further demonstrated by Spitzer non-detections (Marengo et al. 2009; Janson et al. 2012), a perturbing planet is required to account for the observed disk asymmetry (Kalas et al. 2005; Quillen 2006; Chiang et al. 2009). Nevertheless, recent re-analyses on the public $H S T$ data by Currie et al. (2012) and Galicher et al. (2012) confirm the detection of Fomalhaut b, with its position found to be just interior to the ring and comoving with the star, making it a candidate to maintain the sharp inner boundary of the Fomalhaut cold ring. The orbit of Fomalhaut $b$ has not yet been fully demonstrated to be consistent with the range of orbits required for the perturber, and its mass is also quite uncertain due to the lack of detections at other wavelengths, ranging from $<\mathrm{a}$ few $M_{J}$ (from the constraint of cold belt as detected in scattered light; Chiang et al. 2009) to a few $M_{\oplus}$ (from the constraint derived from the properties of the cold belt in the submillimeter; Boley et al. 2012). Furthermore, ground-based high-contrast observations have also set a mass limit of $<2 M_{J}$ for any planet between $\sim 10-40$ AU (Kenworthy et al. 2009) and of $<12-20 M_{J}$ from 4 to 10 AU (M. A. Kenworthy et al. 2012, submitted) in the Fomalhaut system.

The clumpy structures claimed from millimeter and submillimeter imaging of the Vega cold Kuiper-belt analog were often taken as signatures of gravitational perturbations by planets (Holland et al. 1998; Wilner et al. 2002; Wyatt 2003; Marsh et al. 2006). Recent observations at higher resolution and sensitivity have failed to detect the clumps and instead are consistent with a smooth, broad, and axisymmetric disk (Piétu et al. 2011; Hughes et al. 2012). In any case, Vega has been the target of several deep searches for planets through direct imaging, and strong limits have been placed at the $H, L^{\prime}$, and $M$ bands for planets with masses $\gtrsim 3-4 M_{J}$ between $\sim 20$ and $\sim 70$ AU (Marois et al. 2006; Heinze et al. 2008). For $\epsilon$ Eri, planets with masses $\gtrsim 2-3$ $M_{J}$ in a radial distance of 6-35 AU are ruled out at $H, L^{\prime}$, and $M$ bands (Lafrenière et al. 2007; Heinze et al. 2008) and Spitzer IRAC bands (Marengo et al. 2006, 2009). Using radial velocity $(\mathrm{RV})$ and astronometry techniques, a close, eccentric planet, $\epsilon$ Eri b, with a semimajor distance of 3-4 AU was identified by Hatzes et al. (2000) and Benedict et al. (2006), although the discovery of the inner warm dust belt $(\sim 3 \mathrm{AU})$ casts doubt on the existence of a high eccentricity planet (Backman et al. 2009). Furthermore, a recent analysis of all available RV data of $\epsilon$ Eri by Anglada-Escudé \& Butler (2012) found a significantly different orbital solution and suggested that the long-term RV variability is likely due to stellar activity cycles rather than a putative planet. Nonetheless, $\epsilon$ Eri $b$, if it exists just outside the warm belt, is a candidate to shepherd the warm dust belt (Backman et al. 2009).

Although the exact locations of the warm components in these disks are unknown (unresolved), the orbital ratios $\left(R_{\text {cold }} / R_{\text {warm }}\right)$ between the outer cold belt (mostly resolved in the submillimeter and millimeter wavelengths) and the warm belt estimated from the dust temperature are roughly $\gtrsim 10$ in all four systems, ${ }^{13}$

\footnotetext{
13 Vega: $R_{\text {warm }} \sim 14$ AU and $R_{\text {cold }} \sim 110 \mathrm{AU}$; Fomalhaut: $R_{\text {warm }} \sim 10$ AU and $R_{\text {cold }} \sim 140 \mathrm{AU}$; HR 8799: $R_{\text {warm }} \sim 10 \mathrm{AU}$ and $R_{\text {cold }} \sim 100 \mathrm{AU} ; \epsilon$ Eri: $R_{\text {warm }} \sim 3$ AU and $R_{\text {cold }} \sim 35$ AU.
}

similar to the ratio in our own solar system (the asteroid belt at $\sim 3 \mathrm{AU}$ and the Kuiper belt at $\sim 35 \mathrm{AU})$. A significant deficit of large grains (best tracers for the unseen parent bodies) in the region between the belts are evident due to the fact that high-resolution submillimeter observations do not detect such a filled-in component (Boley et al. 2012), although small grains can drift inward from the cold belt (Acke et al. 2012). The zone between the warm and cold belts that is mostly free of dust is very large. If the observed dust is being generated in both belts through collisional cascades of large parent bodies, then we need a cleaning mechanism to maintain such a large dust-free zone.

The large gap between the warm and cold belts may be maintained by one or multiple planet-mass objects in the gap. If these planets have dynamical influence over the entire gap, then they are likely to scatter any material that is either generated in the gap or drifts into it. The dynamical influence of a planet is given approximately by the extent of the overlap of firstorder resonances which creates an unstable "chaotic zone" in the vicinity of a planetary perturber (Wisdom 1980; Duncan et al. 1989; Mustill \& Wyatt 2012). Applying this criterion, we find that in the Vega system, the large gap could be maintained by a single hypothetical object with a mass of a few $100 M_{J}$ (i.e., a star/brown dwarf) in a circular orbit; for Fomalhaut, a similar mass estimate of a few $100 M_{J}$ is obtained for a single hypothetical object in an eccentric orbit $(e=0.1$, based on the observed eccentricity of its cold dust ring). One can also imagine a lower mass perturber in a very eccentric orbit being responsible for maintaining such a large gap; the required eccentricity can be estimated by assuming that its pericenter and apocenter are near the locations of the warm and cold belts. We find that a large eccentricity $(e \sim 0.8)$ is required, implying an object with a mass of $\sim 50 M_{J}$ in both cases. Our simple estimates show that the gaps in both Vega and Fomalhaut are too large to be explained by a single circular/eccentric perturber, without contradicting the current upper limits of a few $M_{J}$ based on non-detections of planetary companions in the gaps.

The minimum number of planets residing between the two belts is two-one inner planet outside the warm dust belt to shepherd the inner planetesimal belt, and one outer planet interior to the cold dust belt to scatter large grains that drift inward from the cold belt. We can then estimate the mass of two equal-mass planets in this case. This is illustrated in Figure 9, where we plot the orbital distances of the two equal-mass planets and their associated chaotic zone widths as a function of the mass ratio between the planet and the host star. In the case of Vega $\left(M_{*}=2.5 M_{\odot}\right.$ and we assume $e=0$ for the hypothetical planets), we find that two $\sim 40 M_{J}$ planets are needed to maintain the dust free zone. In the case of Fomalhaut $\left(M_{*}=2.0 M_{\odot}\right.$ and we assume $e=0.1$ for the hypothetical planets), we find that two $\sim 63 M_{J}$ planets are needed. Although these masses are likely overestimates because the single-planet chaotic zone formulae do not account for the strong secular perturbations that can extend the unstable zones in multiple planet systems (e.g., Moro-Martín et al. 2010), it is evident that just two planets in low eccentricity orbits with mass $\sim M_{J}$ are inadequate for explaining the gaps.

Our simplified approach, estimating the masses for single planetary perturbers and for two equal-mass perturbers in order to explain the large gaps in Vega and Fomalhaut, gives limits that are more than an order of magnitude higher than the $\sim$ few $M_{J}$ planet mass limits for these systems based on non-detections of planetary companions in the gaps. It is, therefore, probable 

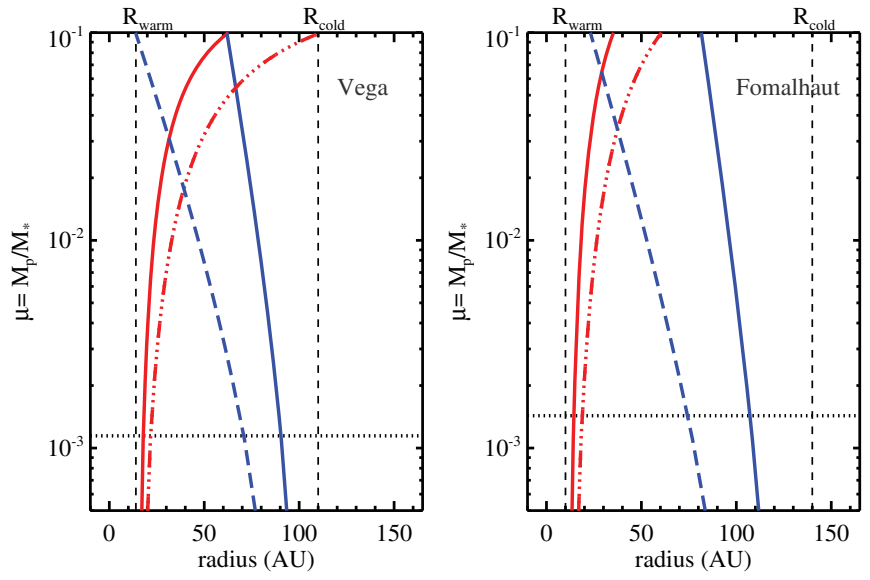

Figure 9. Mass-dependent chaotic zones for two equal-mass planets in the Vega (left panel) and Fomalhaut (right panel) systems, related to the boundaries of the inner and outer belts (shown as black, dashed, vertical lines). In both panels, the orbital radius of the inner planet is shown as red solid lines, while that of the outer planet is shown as blue solid lines. In Vega, the chaotic zone width is computed using $e=0$ for the planet's eccentricity, but for Fomalhaut we adopt $e=0.1$. The dotted-dash lines represent the outer boundary of the chaotic zone for the inner planet while the long-dashed lines represent the inner boundary of the chaotic zone for the outer planet. For two equal-mass planets, the planet-star mass ratio, $\mu$, has to be greater than $0.015(0.03)$ for these two planets to maintain the dust-free zone in the Vega (Fomalhaut) system. The current observational upper limit for the planet mass is shown as the horizontal dotted lines.

(A color version of this figure is available in the online journal.)

that multiple, lower mass planets are responsible for clearing the gaps. We can estimate the numbers and orbital radii of multiple, Jupiter-mass planets that would clear this region by assuming that they are separated such that their chaotic zones just fill the regions between them. We find that four or five $1 M_{J}$ mass planets are required for the large gap in Vega and in Fomalhaut. (This is reminiscent of the HR 8799 system in which four planets separate the two dust belts.) Upcoming groundbased high-contrast, direct imaging surveys using facilities like LBT/LBTI, Gemini/GPI, VLT/SPHERE should be able to find and/or place tighter mass limits on the planets in the large gap. Stars that have a debris disk with two distinct dust belts separated by a large gap are attractive targets for future searches for exoplanets.

\subsection{The Mystery of the Hot Dust}

The presence of hot dust in the close vicinity of both systems is revealed by ground-based interferometric observations. For Fomalhaut, $0.88 \% \pm 0.12 \%$ of excess emission in the $K$ band was reported by Absil et al. (2009). For Vega, a 1.29\% \pm $0.19 \%$ excess in the $K$ band (Absil et al. 2006) and a $1.23 \% \pm$ $0.45 \%$ excess in the $\mathrm{H}$ band (Defrère et al. 2011) were reported. Generally, these studies have ruled out other possible sources such as low-mass companions or stellar winds as the cause of excess emission. Mennesson et al. (2011) also put a very tight constraint on the location of the hot dust (within $0.2 \mathrm{AU}$ ) by combining all interferometric measurements. Defrère et al. (2011) further modeled the properties of the hot excess of Vega (both spatial visibility and SED) and reached several conclusions: the dust responsible for this hot excess (1) has very steep density and particle size distributions (density power index less than -3 and particle size power index $\sim-5$ ), (2) resides from $0.1 \mathrm{AU}$ (dust sublimation radius with temperature of $1700 \mathrm{~K}$ ) to less than $0.2 \mathrm{AU}$ from the star, and (3) has a minimum particle size of $0.01-0.2 \mu \mathrm{m}$ with a significant fraction of carbonaceous composition (due to the fact that carbonaceous grains have a much higher sublimation temperature and lack prominent mineralogical features). Although their model does include large grains (with maximum size of $1000 \mu \mathrm{m}$ ) in the calculation, there is basically no grain with sizes larger than $0.3 \mu \mathrm{m}$ (the average size particle is $0.27 \mu \mathrm{m}$ ) with such a steep size distribution. In other words, the only explanation for such a hot excess is a population of sub- $\mu \mathrm{m}$ carbonaceous grains located in a narrow-ring-like region at the dust sublimation radius $(0.1 \mathrm{AU})$. In the case of Vega, this hot excess is very different from what we know about the zodiacal cloud in our solar system, which has a relatively flat density distribution (density power index of -0.34 ; Kelsall et al. 1998) and mostly contains large particles with sizes of $\sim 10-100 \mu \mathrm{m}$ (Fixsen \& Dwek 2002). The use of exozodi in this context, as has been referred to broadly in the literature for Vega, is misleading.

The origin of the hot dust population is unknown. One scenario that has been discussed frequently in the literature is that the hot dust arises from evaporating comets dynamically perturbed from the outer cold disks. In the case of Vega, a total mass of $\sim 10^{-9} M_{\oplus}$ is required to account for the observed excess emission in near-IR (Defrère et al. 2011). The radiation blow-out timescale at $0.1 \mathrm{AU}$ is on the order of a year, suggesting a very high dust replenishing rate. This high replenishing rate implies that this phenomenon is unlikely to be in a static state. If not in steady-state, then the hot dust may be created in transient dynamical events similar to the late heavy bombardment in our solar system, which was caused by a dynamical instability of the asteroid belt (Strom et al. 2005). However, this kind of hot excess has also been found around stars that have no detectable infrared excess indicative of the presence of a planetesimal population (Absil et al. 2008), making this scenario unsatisfactory to explain the hot excess phenomenon.

Kobayashi et al. (2009) present an analytical model to form a narrow ring due to sublimation of dust grains drifting radially inward due to P-R drag. This scenario could work in Vega and Fomalhaut if the dust drifting inward is refractory, i.e., the asteroid belt discussed in this paper is the source of the particles. This hypothesis only requires a P-R dominated disk; no dynamical perturbation is required. The replenishing rate implies that a total mass of $>0.4 M_{\oplus}$ in the Vega asteroid belt is required to retain such a rate over the age of the system ( $\sim 400 \mathrm{Myr}$ ). However, the range of distances and particle sizes from this drag-in component would result in a much higher excess flux in the mid-IR (see Figure 5 in Kobayashi et al. 2011). Furthermore, one would expect that the amount of hot dust should be proportional to the amount of dust in the source region, i.e., the asteroid belt. The fact that the hot dust in both systems has a similar fractional luminosity, $5 \times 10^{-4}$ (Absil et al. 2006, 2009), while the warm dust in the Fomalhaut system is $\sim 3$ times more than that of the Vega system, argues against this non-stochastic transport hypothesis.

There may be an alternative direction for models of the hot dust component. The basic requirements are (1) a mechanism that prevents the radiation-pressure blow-out of very small dust grains at very small astrocentric distances $(\sim 10$ stellar radii, i.e., the $\sim 1500 \mathrm{~K}$ dust that is creating the $K$-band excess); (2) a grain composition that yields a featureless, roughly Rayleigh-Jeans (or steeper than Rayleigh Jeans) spectrum between 2 and $10 \mu \mathrm{m}$ (for consistency with the SED constraints in Section 3.1); and (3) a mechanism for the generation grains of the appropriate composition. Although a detailed analysis is beyond the scope of this paper, these requirements evoke the behavior of nano dust grains (with sizes of ten to a few tens of $\mathrm{nm}$ ) that are charged by 


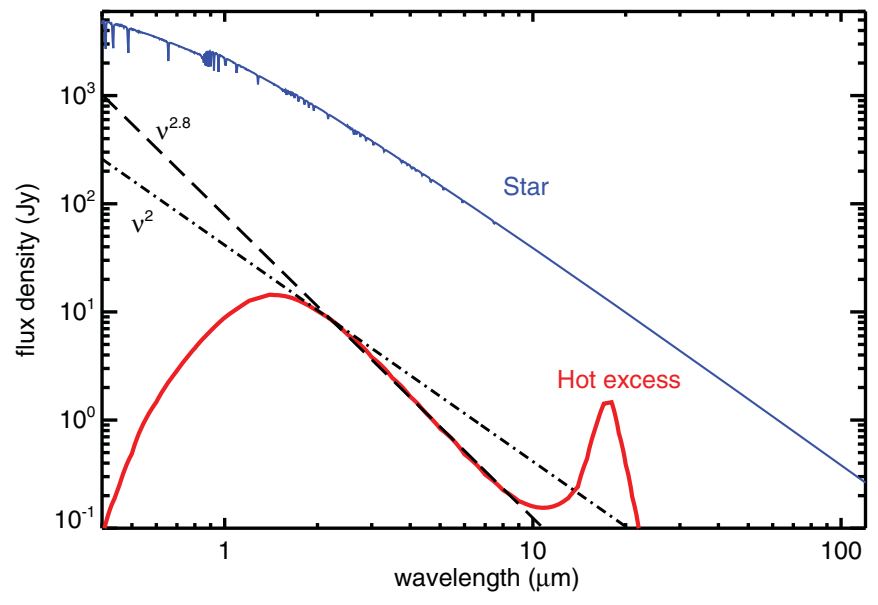

Figure 10. Model SED of the hot excess around Vega using nano oxide particles. Although the stellar spectrum shown on this plot (blue, thin, solid line) is the Kurucz atmospheric model (viewed pole-on), the actual heating spectrum is used the one viewed by the dust along the equator of Vega. The model spectrum (solid red line) and various power-law lines (as frequency $v$ ) are normalized to $2.2 \mu \mathrm{m}$ to account for the $1.29 \%$ excess at $K$ band.

(A color version of this figure is available in the online journal.)

the solar wind and trapped by the solar magnetic field near the Sun. The underlying hypothesis is that sungrazing planetesimals deliver silicate-rich grains to the stellar neighborhood, but when the silicates break down they yield metal oxides (Mann \& Murad 2005). Because of the high abundance of $\mathrm{Mg}$ and Fe in silicates, $\mathrm{MgO}$ and $\mathrm{FeO}$ are produced profusely in this process through reactions such as

$$
\mathrm{MgSiO}_{3} \rightarrow \mathrm{MgO}+\mathrm{SiO}+\frac{1}{2} \mathrm{O}_{2}
$$

and

$$
\mathrm{Fe}_{2} \mathrm{SiO}_{4} \rightarrow 2 \mathrm{FeO}+\mathrm{SiO}+\frac{1}{2} \mathrm{O}_{2} .
$$

The resulting nano grains are very refractory: for example, $\mathrm{MgO}$ has a melting temperature of $3100 \mathrm{~K}$ and boiling temperature of $3873 \mathrm{~K}$, nearly as high as the sublimation temperature of carbon, $3900 \mathrm{~K}$; FeO boils at $3687 \mathrm{~K}$. Consequently, $\mathrm{MgO}, \mathrm{FeO}$, and composite grains of the same elements can survive close to the star. We use the hot excess around Vega as an example to illustrate the resultant SED since the location for such a hot excess is best constrained among all the $K$-band excess sources. We adopted the optical constants for a mixed iron/magnesium oxide, $\mathrm{Mg}_{0.6} \mathrm{Fe}_{0.4} \mathrm{O}$ (Henning et al. 1995) and computed their absorption and scattering efficiencies using the Mie theory. We computed a model SED (Figure 10) for such a narrow ring located between 0.18 and $0.2 \mathrm{AU}$ from the star, and composed of grains with radii of 5-20 nm $(0.005-0.02 \mu \mathrm{m})$ in a size-distribution power index of -3.5 using the stellar model computed by Aufdenberg et al. (2006; for dust viewed from the stellar equator). The dust temperatures for these gains in the region of 0.18-0.2 AU from Vega reach $\sim 2400 \mathrm{~K}$. With a grain density of $4.8 \mathrm{~g} \mathrm{~cm}^{-3}$, a total mass of $2.3 \times 10^{-9} M_{\oplus}$ produces the $1.29 \%$ excess of the star at $2.2 \mu \mathrm{m}$. Toward longer wavelengths to $10 \mu \mathrm{m}$, the output falls roughly as $v^{2.8}$, where Rayleigh-Jeans falls as $v^{2}$, that is, the spectrum is somewhat steeper than Rayleigh-Jeans (see Figure 10). There is a prominent feature at $\sim 18 \mu \mathrm{m}$ in our model SED due to the crystalline form of the material used to determine the optical constants, which might not represent the actual form of material resulting from sublimation of silicate-rich planetesimals. The mass in nano dust is equivalent to about 60 Halley Comet nuclei. Although the conversion of a comet nucleus to nano dust will not be fully efficient, it should be reasonably high since the process involves erosion of large grains, and so most of the solid material (which is believed to constitute more than half the mass of a typical comet nucleus) may be converted into oxides. Thus, it appears that a plausible number of planetesimals (perhaps no more than a couple of hundred) would suffice to provide the nano grain population. Finally, we would like to emphasize that the model SED shown in Figure 10 is one example that satisfies the requirements (as listed previously) in our hypothesis. Our proposed scenario does not depend on the exact nature of the material as long as they meet these requirements.

We now consider the magnetic trapping of these dust grains. Vega has a magnetic field strength of $\sim 1.2 \mathrm{G}$, with about a quarter in a dipolar component and the rest in higher order terms (Petit et al. 2010). To first order, this is similar to that of the Sun, with a surface field of about $1 \mathrm{G}$ dominated by a dipole but with complex components due to activity. Nano grains in the vicinity of Vega will acquire electric charge through the photoelectric effect. This process has been modeled by Pedersen \& Gómez de Castro (2011), who find that a $30 \mathrm{~nm}$ grain illuminated by an A0 star will reach a level of about $200 e^{-}$or charge per mass ratio $(Q / m) \sim 2 \times 10^{-6}$. Smaller grains will reach higher values of $Q / m$. Assuming a field similar to that of the Sun, these values are in the range where trapping occurs (Czechowski \& Mann 2010). In summary, the hypothesis that the $2 \mu \mathrm{m}$ excess is emitted by nano grains trapped in the magnetic field of Vega does satisfy the three conditions listed above. A better understanding of the behavior of the magnetic field of the star is required to improve our understanding of whether it is indeed the process that accounts for this emission. Furthermore, this magnetically trapped, nano dust model can apply to other stars that show $K$-band hot excesses through interferometry as long as these stars possess some magnetic field and can charge these nano particles through the photoelectric effect (for early-type star) or stellar wind (for late-type stars).

\section{CONCLUSIONS}

Nearby debris disks, including the debris disk twins Vega and Fomalhaut, have been playing an important role in our understanding of the underlying planetary architectures (planets and minor bodies) since their first discovery by IRAS through infrared excesses. Much attention has been focused on their large, cold Kuiper-belt-analog rings because they contain the majority of the leftover planetesimals and fine debris that covers a large surface area, making them readily detectable through infrared and submillimeter observations. The Fomalhaut debris system possesses an unresolved warm excess first suggested by the Spitzer resolved image at $24 \mu \mathrm{m}$ (Stapelfeldt et al. 2004). Using the Spitzer IRS spectrum centered at this warm excess along with the photometry measured at the star position from the Herschel $70 \mu \mathrm{m}$ and ALMA $850 \mu \mathrm{m}$ images, we corroborate that the warm $(\sim 170 \mathrm{~K})$ excess arises from thermal dust emission and is very unlikely to originate from stellar wind as suggested by Acke et al. (2012). Through resolved images at multiple wavelengths and mid-infrared spectrum, we identified for the first time the presence of a warm $(\sim 170 \mathrm{~K})$, unresolved component in the Vega debris disk, which is clearly separated from the cold belt. Similar to the one in the Fomalhaut system, we suggest that this warm component also arises from thermal dust emission from a planetesimal belt located near the 
water-frost line, analogous to the asteroid belt in our solar system. The warm belts in both systems share many similar characteristics. No extension at both MIPS 24 and PACS $70 \mu \mathrm{m}$ (angular radius $\left.\lesssim 3^{\prime \prime}\right)$ and the observed dust temperature $(\sim 170 \mathrm{~K})$ place this warm belt at $\sim 2^{\prime \prime}$ from Vega and $\sim 1^{\prime \prime} .5$ from Fomalhaut for blackbody-like grains. Furthermore, the stringent constraints on the excess levels from 3 to $6 \mu \mathrm{m}$ around both stars indicate that this warm belt is not spatially associated with the $2 \mu \mathrm{m}(K-$ band) excesses inferred from the interferometric observations.

Including the debris disks around $\epsilon$ Eri and HR 8799, the four nearby debris systems all have spatially separated warm and cold belts where the observed dust temperatures for the warm belt are at $\sim 150-170 \mathrm{~K}$, whereas the cold belt temperatures are at $\sim 45-50 \mathrm{~K}$ despite the stark differences in stellar luminosity. Similar bi-modal dust temperature structure is also found in other debris disk systems. Systems where the location of the cold belts is known through resolved images, either in scattered light or thermal infrared/submillimeter wavelengths, and the association of a separate warm belt is inferred from spectrophotometric mid-infrared excesses include the following: HD 10647, HD 15115, HD 107146, and HD 139664 (although not all of them have the warm-belt temperature at $\sim 150 \mathrm{~K}$ ). Bi-modal temperature distributions inferred from SED analysis for many other unresolved sources (Morales et al. 2011) argue that the debris disk structures are, somehow, determined by temperaturesensitive processes. It is well known that the location of the water-frost line in protoplanetary disks plays an important role in the formation of planetesimals. An asteroid belt located near the frost line for these warm belts seems to imply that they are inherited from the early evolution of planetary systems.

The $K$-band excesses have been found around roughly a dozen nearby main-sequence stars using ground-based interferometric techniques (Absil et al. 2006; di Folco et al. 2007; Absil et al. 2008, 2009; Akeson et al. 2009). In some cases, sources like low-mass companions, ionized stellar wind, or stellar scattered light have been ruled out except for where hot $(\sim 1500 \mathrm{~K})$ dust emission is responsible for the excess emission. Recent $N$-body simulations done by Bonsor et al. (2012) have demonstrated the difficulty of scattering enough small bodies inward by a chain of planets inside a cold outer belt to sustain the observed level of small grains in Vega and $\eta$ Crovi. In the case of Vega, the positive detections and constraints from non-detections using different interferometric facilities and wavelengths affirm the presence of very small grains in the very close vicinity $(<0.2 \mathrm{AU})$ around the star. The properties of this hot dust are very different from what we know about the particles in our own zodiacal cloud; instead, they are more likely to arise from the phenomenon observed near the Sun where nano particles are trapped in the magnetic field of the star. In our proposed scenario, nano-size metal oxides originate from the sublimation of silicate-rich planetesimals, and are charged either via the photoelectric effect or the stellar wind, and then are magnetically trapped in close proximity to the star. The replenishing rate of these tiny particles can be very low once they are trapped. Thus, our scenario does not require a massive reservoir of leftover planetesimals; therefore, it presumably works for stars that show hot $2 \mu \mathrm{m}$ excess but without detectable cold dust.

Although the warm belts in the four systems discussed in this paper are not directly resolved, unlike the cold belts, the orbital ratios between the outer cold and inner warm belts are roughly $\gtrsim 10$ based on the observed dust temperatures. The large gap between the two planetesimal belts requires a mechanism to maintain it mostly free of dust. The most plausible explanation is the existence of planetary bodies in the gaps, analogous to HR 8799 and our own solar system. From simple chaotic zone calculations and the mass limit on Fomalhaut $\mathrm{b}$, we argue that multiple low-mass $\left(\lesssim 1 M_{J}\right)$ planets are required to maintain such a large gap. A similar argument is also supported for the Vega system, suggesting that the widely separated, two-belt debris systems are signposts for the presence of multiple low-mass planets. Our results are in line with the recent result by Wyatt et al. (2012), where a positive correlation is identified between the debris disk detection rate and the presence of exoplanets with masses less than Saturn around 60 nearby G-type stars, and echo the recent RV and Kepler results (Mayor et al. 2011; Batalha et al. 2012) that low-mass planets are more common than the massive ones, and that multiple planet systems are rather common among exoplanetary systems.

We thank the anonymous referee for prompt and constructive comments. K.Y.L.S. thanks Denis Defrére for his helpful discussion of the hot excesses. This work is based on observations made with the Spitzer Space Telescope, which is operated by the Jet Propulsion Laboratory, California Institute of Technology. K.Y.L.S. and K.R.S. are grateful for funding from NASA's ADAP program (grant No. NNX11AF73G). Support for G.H.R. is provided by NASA through contracts 1255094 and 1256424 issued by JPL/Caltech to the University of Arizona. A.M.H. is supported by a fellowship from the Miller Institute for Basic Research in Science. A.B. is supported by the ANR-2010 BLAN-0505-01 (EXEXOZODI) program. Z.B. is funded by the Deutsches Zentrum für Luf- und Raumfahrt (DLR). Partial support for this work was also provided for Z.B. through Hungarian OTKA grant No. K81966.

\section{REFERENCES}

Absil, O., di Folco, E., Mérand, A., et al. 2006, A\&A, 452, 237 Absil, O., di Folco, E., Mérand, A., et al. 2008, A\&A, 487, 1041 Absil, O., Mennesson, B., Le Bouquin, J.-B., et al. 2009, ApJ, 704, 150 Acke, B., Min, M., Dominik, C., et al. 2012, A\&A, 540, A125

Akeson, R. L., Ciardi, D. R., Millan-Gabet, R., et al. 2009, ApJ, 691, 1896 Anglada-Escudé, G., \& Butler, R. P. 2012, ApJS, 200, 15

Aufdenberg, J. P., Mérand, A., Coudé du Foresto, V., et al. 2006, ApJ, 645, 664 Backman, D., Marengo, M., Stapelfeldt, K., et al. 2009, ApJ, 690, 1522

Batalha, N. M., Rowe, J. F., Bryson, S. T., et al. 2012, ApJS, submitted (arXiv: 1202.5852)

Benedict, G. F., McArthur, B. E., Gatewood, G., et al. 2006, AJ, 132, 2206

Bonsor, A., Augereau, J.-C., \& Thebault, P. 2012, A\&A, 548, 104 Boley, A. C., Payne, M. J., Corder, S., et al. 2012, ApJL, 750, 21

Chen, C. H., Sheehan, P., Watson, D. M., Manoj, P., \& Najita, J. R. 2009, ApJ, 701, 1367

Chiang, E., Kite, E., Kalas, P., Graham, J. R., \& Clampin, M. 2009, ApJ, 693,734

Currie, T., Debes, J., Rodigas, T. J., et al. 2012, ApJL, 760, 32

Czechowski, A., \& Mann, I. 2010, ApJ, 714, 89

Defrère, D., Absil, O., Augereau, J.-C., et al. 2011, A\&A, 534, A5

Dermott, S. F., Jayaraman, S., Xu, Y. L., Gustafson, B. Å. S., \& Liou, J. C. 1994, Natur, 369, 719

Dermott, S. F., Kehoe, T. J. J., Durda, D. D., Grogan, K., \& Nesvorný, D. 2002, in Asteroids, Comets, and Meteors: ACM 2002, ed. B. Warmbein (ESA SP-500; Noordwijk, Netherlands: ESA Publications Division), 319

di Folco, E., Absil, O., Augereau, J.-C., et al. 2007, A\&A, 475, 243

Dominik, C., \& Decin, G. 2003, ApJ, 598, 626

Duncan, M., Quinn, T., \& Tremaine, S. 1989, Icar, 82, 402

Fixsen, D. J., \& Dwek, E. 2002, ApJ, 578, 1009

Galicher, R., Marois, C., Zuckerman, B., \& Macintosh, B. 2012, arXiv: 1210.6745

Gáspár, A., Psaltis, D., Rieke, G. H., \& Ozel, F. 2012, ApJ, 754, 74

Hatzes, A. P., Cochran, W. D., McArthur, B., et al. 2000, ApJL, 544, 145

Heinrichsen, I., Walker, H. J., \& Klaas, U. 1998, MNRAS, 293, L78

Heinze, A. N., Hinz, P. M., Kenworthy, M., Miller, D., \& Sivanandam, S. 2008, ApJ, 688, 583 
Higdon, S. J. U., Devost, D., Higdon, J. L., et al. 2004, PASP, 116, 975

Henning, T., Begemann, B., Mutschke, H., \& Dorschner, J. 1995, A\&AS, 112,143

Holland, W. S., Greaves, J. S., Zuckerman, B., et al. 1998, Natur, 392, 788

Hughes, A. M., Wilner, D. J., Mason, B., et al. 2012, ApJ, 750, 82

Janson, M., Carson, J. C., Lafrenière, D., et al. 2012, ApJ, 747, 116

Johnson, J. A., Aller, K. M., Howard, A. W., \& Crepp, J. R. 2010, PASP, 122,905

Kalas, P., Graham, J. R., Chiang, E., et al. 2008, Sci, 322, 1345

Kalas, P., Graham, J. R., \& Clampin, M. 2005, Natur, 435, 1067

Kelsall, T., Weiland, J. L., Franz, B. A., et al. 1998, ApJ, 508, 44

Kennedy, G. M., Wyatt, M. C., Sibthorpe, B., et al. 2012, MNRAS, 426, 2115

Kenworthy, M. A., Mamajek, E. E., Hinz, P. M., et al. 2009, ApJ, 697, 1928

Kenworthy, M. A., Meshkat, T., Quanz, S. P., et al. 2012, ApJ, arXiv:1212.1459

Kobayashi, H., Kimura, H., Watanabe, S.-i., Yamamoto, T., \& Müller, S. 2011, EP\&S, 63, 1067

Kobayashi, H., Watanabe, S.-I., Kimura, H., \& Yamamoto, T. 2009, Icar, 201, 395

Kretke, K. A., \& Lin, D. N. C. 2007, ApJL, 664, 55

Lafrenière, D., Doyon, R., Marois, C., et al. 2007, ApJ, 670, 1367

Laor, A., \& Draine, B. T. 1993, ApJ, 402, 441

Liou, J.-C., \& Zook, H. A. 1999, AJ, 118, 580

Liu, W. M., Hinz, P. M., Hoffmann, W. F., et al. 2004, ApJL, 610, 125

Mann, I., \& Murad, E. 2005, ApJL, 624, 125

Marengo, M., Megeath, S. T., Fazio, G. G., et al. 2006, ApJ, 647, 1437

Marengo, M., Stapelfeldt, K., Werner, M. W., et al. 2009, ApJ, 700, 1647

Marois, C., Lafrenière, D., Doyon, R., Macintosh, B., \& Nadeau, D. 2006, ApJ, 641, 556

Marois, C., Macintosh, B., Barman, T., et al. 2008, Sci, 322, 1348

Marois, C., Zuckerman, B., Konopacky, Q. M., Macintosh, B., \& Barman, T. 2010, Natur, 468, 1080

Marsh, K. A., Dowell, C. D., Velusamy, T., Grogan, K., \& Beichman, C. A. 2006, ApJL, 646, 77

Mayor, M., Marmier, M., Lovis, C., et al. 2011, A\&A, submitted (arXiv:1109.2497)

Mennesson, B., Hanot, C., Serabyn, E., et al. 2011, ApJ, 743, 178
Morales, F. Y., Rieke, G. H., Werner, M. W., et al. 2011, ApJL, 730, 29

Moro-Martín, A., Malhotra, R., Bryden, G., et al. 2010, ApJ, 717, 1123

Müller, T., Nielbock, M., Balog, Z., Klaas, U., \& Vilenius, E. 2011,

PACS Photometry-Point-Source Flux Calibration, PICC-ME-TN-037, https://nhscsci.ipac.caltech.edu/sc/index.php/Pacs/AbsoluteCalibration

Murray, N., Holman, M., \& Potter, M. 1998, AJ, 116, 2583

Mustill, A. J., \& Wyatt, M. C. 2012, MNRAS, 419, 3074

Natta, A., Grinin, V., \& Mannings, V. 2000, Protostars and Planets IV (Tucson, AZ: Univ. Arizona Press), 559

Nesvorný, D., Jenniskens, P., Levison, H. F., et al. 2010, ApJ, 713, 816

Ott, S. 2010, adass XIX, 434, 139

Pedersen, A., \& Gómez de Castro, A. I. 2011, ApJ, 740, 77

Petit, P., Lignières, F., Wade, G. A., et al. 2010, A\&A, 523, A41

Piétu, V., di Folco, E., Guilloteau, S., Gueth, F., \& Cox, P. 2011, A\&A, 531, L2

Price, S. D., Paxson, C., Engelke, C., \& Murdock, T. L. 2004, AJ, 128, 889

Price, S. D., Smith, B. J., Kuchar, T. A., Mizuno, D. R., \& Kraemer, K. E. 2010, ApJS, 190, 203

Quillen, A. C. 2006, MNRAS, 372, L14

Reidemeister, M., Krivov, A. V., Stark, C. C., et al. 2011, A\&A, 527, A57

Rieke, G. H., Blaylock, M., Decin, L., et al. 2008, AJ, 135, 2245

Sibthorpe, B., Vandenbussche, B., Greaves, J. S., et al. 2010, A\&A, 518, L130

Stapelfeldt, K. R., Holmes, E. K., Chen, C., et al. 2004, ApJS, 154, 458

Strom, R. G., Malhotra, R., Ito, T., Yoshida, F., \& Kring, D. A. 2005, Sci, 309, 1847

Su, K. Y. L., Rieke, G. H., Misselt, K. A., et al. 2005, ApJ, 628, 487

Su, K. Y. L., Rieke, G. H., Stapelfeldt, K. R., et al. 2009, ApJ, 705, 314

Tokunaga, A. T. 1984, AJ, 89, 172

van Leeuwen, F. 2007, A\&A, 474, 653

Williams, J. P., \& Cieza, L. A. 2011, ARA\&A, 49, 67

Wilner, D. J., Holman, M. J., Kuchner, M. J., \& Ho, P. T. P. 2002, ApJL, 569, 115

Wisdom, J. 1980, AJ, 85, 1122

Wyatt, M. C. 2003, ApJ, 598, 1321

Wyatt, M. C. 2008, ARA\&A, 46, 339

Wyatt, M. C., \& Dent, W. R. F. 2002, MNRAS, 334, 589

Wyatt, M. C., Kennedy, G., Sibthorpe, B., et al. 2012, MNRAS, 424, 1206

Wyatt, M. C., Smith, R., Greaves, J. S., et al. 2007, ApJ, 658, 569 